\title{
A New Weight Balanced Binary Search Tree*
}

\author{
Seonghun $\mathrm{Cho}^{\dagger} \quad$ Sartaj Sahni ${ }^{\dagger}$
}

\begin{abstract}
We develop a new class of weight balanced binary search trees called $\beta$-balanced binary search trees $(\beta$-BBSTs). $\beta$-BBSTs are designed to have reduced internal path length. As a result, they are expected to exhibit good search time characteristics. Individual search, insert, and delete operations in an $n$ node $\beta$-BBST take $\mathrm{O}(\log n)$ time for $0<\beta \leq \sqrt{2}-1$. Experimental results comparing the performance of $\beta$-BBSTs, $\mathrm{WB}(\alpha)$ trees, AVL-trees, red/black trees, treaps, deterministic skip lists and skip lists are presented. Two simplified versions of $\beta$-BBSTs are also developed.
\end{abstract}

Keywords and Phrases. data structures, weight balanced binary search trees

\section{Introduction}

A dictionary is a set of elements on which the operations of search, insert, and delete are performed. Many data structures have been proposed for the efficient representation of a dictionary [SAHN98]. These include direct addressing schemes such as hash tables and comparison schemes such as binary search trees, AVL-trees [AVL62], red/black trees [GUIB78], trees of bounded balance [NIEV73], treaps [ARAG89], deterministic skip lists [MUNR92], and skip lists [PUGH90]. Of these schemes, AVL-trees, red/black trees, and trees of bounded balance $(\mathrm{WB}(\alpha))$ are balanced binary search trees. When representing a dictionary with $n$ elements, using one of these schemes, the corresponding binary search tree has height $O(\log n)$ and individual search, insert, and delete operations take $\mathrm{O}(\log n)$ time. When (unbalanced) binary search trees, treaps, or skip lists are used, each operation has an expected complexity of $\mathrm{O}(\log n)$ but the worst case complexity is $\mathrm{O}(n)$. When hash tables are used, the expected complexity is $\mathrm{O}(1)$ per operation. However, the worst case complexity is $\mathrm{O}(n)$. So, in applications where a worst case complexity guarantee is critical, one of the balanced binary

*This research was supported, in part, by the Army Research Office under grant DAA H04-95-1-0111, and by the National Science Foundation under grant MIP91-03379.

${ }^{\dagger}$ Department of Computer and Information Science and Engineering, University of Florida, Gainesville, FL 32611, U.S.A. 
search tree schemes is to be performed. In applications such as best-fit bin packing we wish to find the smallest element that is greater than or equal to given value (see [SAHN98] page 543). We call this a proximity search. Proximity searches may be done in $\mathrm{O}(\log n)$ time using balanced search trees. These searches take $\mathrm{O}(n)$ time when a hash table is used. Further, indexed versions of balanced search trees may be used to perform linear list operations such as insert and delete in $\mathrm{O}(\log n)$ time (see [SAHN98] page 509, Exercise 21). Other linear list representations such as arrays and chains take $\mathrm{O}(\log n)$ time for these operations.

In this paper, we develop a new weight balanced binary search tree called $\beta$-BBST ( $\beta$ balanced binary search tree). Like $\mathrm{WB}(\alpha)$ trees, this achieves balancing by controlling the relative number of nodes in each subtree. Therefore, like $\mathrm{WB}(\alpha)$ trees $\beta$-BBST trees incur a per node space overhead equal to the space needed to store the weight of a subtree (typically a 4 byte int is used for this purpose). However, unlike $\mathrm{WB}(\alpha)$ trees, during insert and delete operations, rotations are performed along the search path whenever they reduce the internal path length of the tree (rather than only when a subtree is out of balance). As a result, the constructed trees are expected to have a smaller internal path length than the corresponding $\mathrm{WB}(\alpha)$ tree. When dictionary elements are searched for with equal probability, the average search time is closely related to the internal path length. Therefore, the time need to search in a $\beta$-BBST is expected to be less than that in a $\mathrm{WB}(\alpha)$ tree.

In Section 2, we define the total search cost of a binary search tree and show that the rebalancing rotations performed in AVL and red/black trees might increase this metric. We also show that while similar rotations in $\mathrm{WB}(\alpha)$ trees do not increase this metric, insert and delete operations in $\mathrm{WB}(\alpha)$ trees do not avail of all opportunities to reduce the metric. In Section 3, we define $\beta$-BBSTs and show their relationship to $\mathrm{WB}(\alpha)$ trees. Search, insert, and delete algorithms for $\beta$-BBSTs are developed in Section 4. A simplified version of $\beta$ BBSTs is developed in Section 5. Search, insert and delete operations for this version also take $\mathrm{O}(\log n)$ time each. An even simpler version of $\beta$-BBSTs is developed in Section 6 . For this version, we show that the average cost of an insert and search operation is $\mathrm{O}(\log n)$ provided no deletes are performed.

An experimental evaluation of $\beta$-BBSTs and competing schemes for dictionaries (AVL, red/black, skip lists, etc.) was done and the results of this are presented in Section 7. This section also compares the relative performance of $\beta$-BBSTs and the two simplified versions of Sections 5 and 6. 

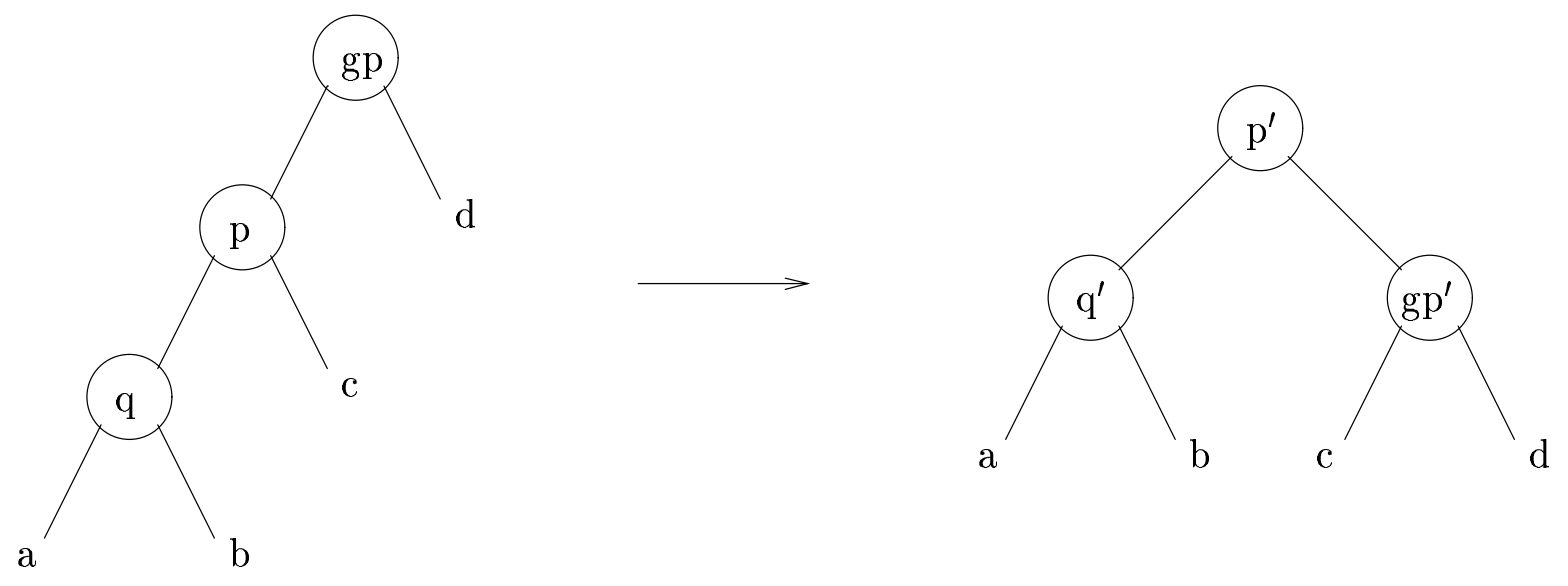

(a) LL rotation
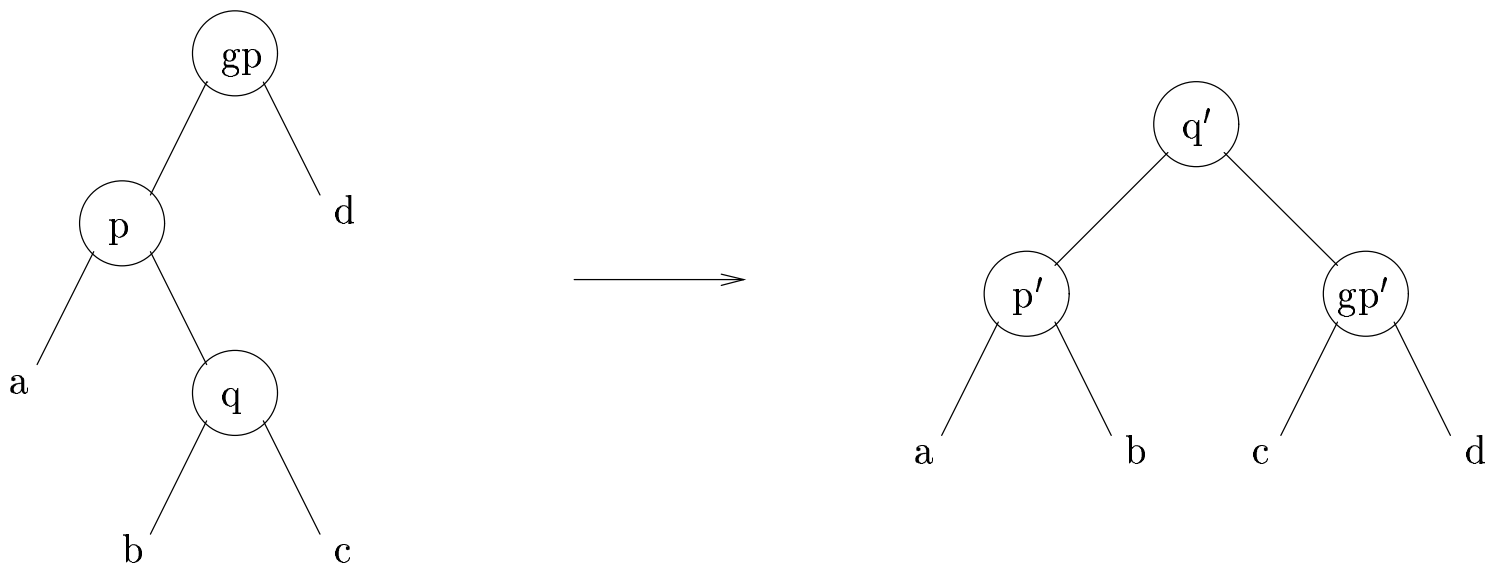

(b) LR rotation

Figure 1: LL (single) and RL (double) rotations

\section{Balanced Trees and Rotations}

Following an insert or delete operation in a balanced binary search tree (e.g., AVL, red/black, $\mathrm{WB}(\alpha)$, etc.), it may be necessary to perform rotations to restore balance. The rotations are classified as LL, RR, LR, and RL [SAHN98]. LL and RR rotations as well as LR and RL rotations are symmetric. While the conditions under which the rotations are performed vary with the class of balanced tree considered, the node movement patterns are the same. Figure 1 shows the transformation performed by an LL and an LR rotation. In this figure, nodes whose subtrees have changed as a result of the rotation are designated by a prime. So, $p^{\prime}$ is the original node $p$ however its subtrees are different. 
Let $h(x)$ be the height of the subtree with root $x$. Let $s(x)$ be the number of nodes in this subtree. When searching for an element $x, x$ is compared with one element at each of $l(x)$ levels, where $l(x)$ is the level at which $x$ is present (the root is at level 1 ). So, one measure of the "goodness" of the binary search tree, $T$, for search operations (assuming each element is searched for with equal probability) is its total search cost defined as:

$$
C(T)=\sum_{x \in T} l(x) .
$$

Notice that $C(T)=I(T)+n$ where $I(T)$ is the internal path length of $T$ and $n$ is the number of elements/nodes in $T$. The cost of unsuccessful searches is equal to the external path length $E(T)$. Since $E(T)=I(T)+2 n$, minimizing $C(T)$ also minimizes $E(T)$.

Total search cost is important as this is the dominant operation in a dictionary (note that insert can be modeled as an unsuccessful search followed by the insertion of a node at the point where the search terminated and deletion can be modeled by a successful search followed by a physical deletion; both operations are then followed by a rebalancing/restructuring step).

Observe that in typical implementations of the search operation in programming languages such as $\mathrm{C}++, \mathrm{C}$, and Pascal, the search for an $x$ at level $l(x)$ will involve up to two comparisons at levels $1,2, \ldots, l(x)$. If the code first checks $x=e_{i}$ where $e_{i}$ is the element at level $i$ to be compared and then $x<e_{i}$ to decide whether to move to the left or right subtree, then the number of element comparisons is exactly $2 l(x)-1$. In this case, the total number of element comparisons is

$$
N C(T)=2 \sum_{x \in T} l(x)-n=2 C(T)-n
$$

and minimizing $C(T)$ also minimizes $N C(T)$. If the code first checks $x<e_{i}$ and then $x=e_{i}$ (or $>e_{i}$ ), the number of element comparisons done to find $x$ is $l(x)+r(x)+1$ where $r(x)$ is the number of right branches on the path from the root to $x$. The total number of comparisons is bounded by $2 C(T)$. An alternative implementation ${ }^{1}$ performs only $<$ compares on the way down. Following the last compare an = compare is done at the last node where a right child move was made. When this scheme is used every search follows a path from the root all the way to a leaf. The worst case number of comparisons for this scheme equals the tree height plus one. When this alternative implementation is used

$$
N C(T)=\sum_{x \text { is a leaf of } T}(r b(x)+1) * l(x)
$$

\footnotetext{
${ }^{1}$ This was pointed out to us by an anonymous referee.
} 
where $r b(x)$ is the number of right branches from the tree root to the leaf $x$. In this case $N C(T)$ does not have a nice relationship to $C(T)$.

For simplicity, we use $C(T)$ to motivate our data structure.

In an AVL tree, when an LL rotation is performed, $h(q)=h(c)+1=h(d)+1$ (see Figure $1(\mathrm{a}))$. At this time, the balance factor at $g p$ is $h(p)-h(d)=2$. The rotation restores height balance which is necessary to guarantee $\mathrm{O}(\log n)$ search, insert, delete operations in an $n$ node AVL tree. The rotation may, however, increase the total search cost. To see this, notice that an LL rotation affects the level numbers of only those nodes that are in the subtree with root $g p$ prior to the rotation. We see that $l\left(q^{\prime}\right)=l(q)-1, l\left(p^{\prime}\right)=l(p)-1, l\left(g p^{\prime}\right)=$ $l(g p)+1$, the total search cost of the subtree with root $a$ is decreased by $s(a)$ as a result of the rotation, etc. Hence, the increase in $C(T)$ due to the rotation is:

$$
\begin{gathered}
l\left(p^{\prime}\right)-l(p)+l\left(q^{\prime}\right)-l(q)+l\left(g p^{\prime}\right)-l(g p)-s(a)-s(b)+s(d) \\
=-1-1+1-s(q)+1+s(d)=s(d)-s(q) .
\end{gathered}
$$

A similar analysis shows that an LR rotation increases $C(T)$ by $s(d)-s(q)$.

If the LL rotation was triggered by an insertion, $s(q)$ is at least one more than the minimum number of nodes in an AVL tree of height $t=h(q)-1$. So, $s(q) \geq \phi^{t+2} / \sqrt{5}$ where $\phi=(1+\sqrt{5}) / 2$. The maximum value for $s(d)$ is $2^{t}-1$. So, an LL rotation has the potential of increasing total search cost by as much as

$$
2^{t}-1-\phi^{t+2} / \sqrt{5} \approx 2^{t}-1-1.62^{t+2} / 2.24 \text {. }
$$

This is negative for $t \leq 2$ and positive for $t>2$. When $t=10$, for example, an LL rotation may increase total search cost by as much as 877. As $t$ gets larger, the potential increase in search cost gets much greater. This analysis is easily extended to the remaining rotations and also to red/black trees. It is interesting to note that the very rotations that are essential to maintaining tree balance may actually increase the search cost.

Definition (WB $(\alpha)$ [NIEV73]) The balance, $B(p)$, of a node $p$ in a binary tree is the ratio $(s(l)+1) /(s(p)+1)$ where $l$ is the left child of $p$. For $\alpha \in[0,1 / 2]$, a binary tree $\mathrm{T}$ is in $\mathrm{WB}(\alpha)$ iff $\alpha \leq B(p) \leq 1-\alpha$ for every node $p$ in T. By definition, the empty tree is in $\mathrm{WB}(\alpha)$ for all $\alpha$.

Lemma 1 (1) The maximum height, $h \max (n)$, of an $n$ node tree in $W B(\alpha)$ is $\sim \log _{\frac{1}{1-\alpha}}(n+$ 1) $[N I E V 73]$

(2) Inserts and deletes can be performed in an $n$ node tree in $W B(\alpha)$ in $O(\log n)$ time for $2 / 11<\alpha \leq 1-\sqrt{2} / 2$ [BLUM80].

(3) Each search operation in an $n$ node tree in $W B(\alpha)$ takes $O(\log n)$ time [NIEVY3]. 
In the case of weight balanced trees $\mathrm{WB}(\alpha)$, an LL rotation is performed when $B(g p) \approx$ $1-\alpha$ and $B(p) \geq \alpha /(1-\alpha)$ (see Figure 1(a)) [NIEV73]. So,

$$
1-\alpha \approx \frac{s(p)+1}{s(g p)+1}=\frac{s(p)+1}{s(p)+s(d)+2}
$$

or

$$
s(d) \approx s(p) \frac{\alpha}{1-\alpha}+\frac{2 \alpha-1}{1-\alpha}
$$

and

$$
\frac{\alpha}{1-\alpha} \leq B(p)=\frac{s(q)+1}{s(p)+1}
$$

or

$$
s(q) \geq s(p) \frac{\alpha}{1-\alpha}+\frac{2 \alpha-1}{1-\alpha} .
$$

So, LL rotations (and also RR) do not increase the search cost. For LR rotations [NIEV73], $B(g p) \approx 1-\alpha$ and $B(p)<\alpha /(1-\alpha)$. So, $s(d) \approx s(p) \frac{\alpha}{1-\alpha}+\frac{2 \alpha-1}{1-\alpha}$ and with respect to Figure $1(\mathrm{~b})$,

$$
\frac{\alpha}{1-\alpha}>B(p)=\frac{s(p)-s(q)}{s(p)+1}
$$

or

$$
s(q)>s(p) \frac{1-2 \alpha}{1-\alpha}-\frac{\alpha}{1-\alpha} .
$$

For $\alpha \leq 1 / 3, s(q) \geq s(d)$ and LR (RL) rotations do not increase search cost. Thus, in the case of $\mathrm{WB}(\alpha)$ trees, the rebalancing rotations do not increase search cost. This statement remains true if the conditions for LL and LR rotation are changed to those in [BLUM80].

While rotations do not increase the search cost of $\mathrm{WB}(\alpha)$ trees, these trees miss performing some rotations that would reduce search cost. For example, it is possible to have

$\alpha<B(g p)<1-\alpha, B(p) \geq \frac{\alpha}{1-\alpha}$, and $s(q)>s(d)$. Since $B(g p)$ isn't high enough, an LL rotation isn't performed. Yet, performing such a rotation would reduce search cost.

\section{$3 \beta$-BBSTs}

Definition A cost optimized search tree (COST) is a binary search tree whose search cost cannot be reduced by performing a single LL, RR, LR, or RL rotation.

Theorem 1 If $T$ is a COST with $n$ nodes, its height is at most $\log _{\phi}(\sqrt{5}(n+1))-2$. This bound is asymptotically achievable.

Proof Let $N_{h}$ be the minimum number of nodes in a COST of height $h$. Clearly, $N_{0}=0$ and $N_{1}=1$. Consider a COST $Q$ of height $h \geq 2$ having the minimum number of nodes $N_{h}$. 
$Q$ has one subtree $R$ whose height is $h-1$ and another, $S$, whose height is $\leq h-1$. $R$ must be a minimal COST of height $h-1$ and so has $N_{h-1}$ nodes. $R$, in return, must have one subtree, $U$, of height $h-2$ and another, $V$, of height $\leq h-2$. Both $U$ and $V$ are COSTs as $R$ is a COST. Since $R$ is a minimal COST, $U$ is a minimal COST of height $h-2$ and so has $N_{h-2}$ nodes. Since $Q$ is a COST, $|S| \geq \max \{|U|,|V|\}$. We may assume that $N_{h}$ is a nondecreasing function of $h$. So, $|S| \geq N_{h-2}$. Since $Q$ is a minimal COST of height $h,|S|=N_{h-2}$. So, $N_{h}=N_{h-1}+N_{h-2}+1, h \geq 2$ and $N_{0}=0, N_{1}=1$. This recurrence is the same as that for the minimum number of nodes in an AVL tree of height $h$. So, $N_{h}=F_{h+2}-1$ where $F_{i}$ is the $i$ 'th Fibbonacci number. Consequently, $N_{h} \approx \phi^{h+2} / \sqrt{5}-1$ and $h \leq \log _{\phi}(\sqrt{5}(n+1))-2$.

Since the height of an AVL tree with $n$ nodes is at $\operatorname{most}_{\log _{\phi}}(\sqrt{5}(n+1))-2 \approx 1.44 \log _{2}(n+$ 2 ), the maximum height of a COST with $n$ nodes is the same as the maximum height of an AVL tree with this many nodes. The worst-case height of a red-black tree with $n$ nodes is $2 \log _{2} n+2$. Therefore, the worst-case height of red-black trees is approximately $40 \%$ more than the worst-case height of AVL trees and COSTs.

Definition Let $a$ and $b$ be the roots of two binary trees. $a$ and $b$ are $\beta$-balanced, $0 \leq \beta \leq 1$, with respect to one another, denoted $\beta$ - $(a, b)$, iff (a) $\beta(s(a)-1) \leq s(b)$ and (b) $\beta(s(b)-1) \leq s(a)$. A binary tree $\mathrm{T}$ is $\beta$-balanced iff the children of every node in $\mathrm{T}$ are $\beta$-balanced.

A full binary tree is 1-balanced and a binary tree whose height equals its size (i.e., number of nodes) is 0-balanced.

Lemma 2 If the binary tree $T$ is $\beta$-balanced, then it is $\gamma$-balanced for $0 \leq \gamma \leq \beta$.

Proof Follows from the definition of balance.

Lemma 3 If the binary tree $T$ is $\beta$-balanced, $0 \leq \beta \leq 1 / 2$, then it is in $W B(\alpha)$ for $\alpha=$ $\beta /(1+\beta)$.

Proof Consider any node $p$ in $T$. Let $l$ and $r$ be node $p$ 's left and right children.

$$
B(p)=\frac{s(l)+1}{s(l)+s(r)+2}=\frac{1}{1+\frac{s(r)+1}{s(l)+1}} .
$$

Since $T$ is $\beta$-balanced, $s(l)-1 \leq s(r) / \beta$ or $s(l)+1 \leq s(r) / \beta+2$. So,

$$
\frac{s(l)+1}{s(r)+1} \leq 1 / \beta+\frac{2 \beta-1}{\beta(s(r)+1)} \leq 1 / \beta
$$


or

$$
\frac{s(r)+1}{s(l)+1} \geq \beta .
$$

So, $B(p) \leq 1 /(1+\beta)$. Further, $s(r)-1 \leq s(l) / \beta$. So,

$$
\frac{s(r)+1}{s(l)+1} \leq 1 / \beta
$$

And, $B(p) \geq 1 /(1+1 / \beta)=\beta /(1+\beta)$. Hence $\beta /(1+\beta) \leq B(p) \leq 1 /(1+\beta)$ for every $p$ in $T$. So, $T$ is in $\mathrm{WB}(\alpha)$ for $\alpha=\beta /(1+\beta)$.

Remark While every $\beta$-balanced tree, $0 \leq \beta \leq 1 / 2$, is in $\mathrm{WB}(\alpha)$ for $\alpha=\beta /(1+\beta)$, there are trees in $\mathrm{WB}(\alpha)$ that are not $\beta$-balanced.

Lemma 4 If $T$ is a COST then $T$ is $\frac{1}{2}$-balanced.

Proof If $T$ is a COST, then every subtree of $T$ is a COST. Consider any subtree with root $p$, left child $l$, and right child $r$. If neither $l$ nor $r$ exist, then $s(l)=s(r)=0$ and $p$ is $\frac{1}{2}$-balanced. If $s(l)=0$ and $s(r)>1$, then $r$ has a nonempty subtree with root $t$ and $s(t)>s(l)$. So $p$ is not a COST. Hence, $s(r) \leq 1$ and $p$ is $\frac{1}{2}$-balanced. The same is true when $s(r)=0$. So, assume $s(l)>0$ and $s(r)>0$.

If $s(l)=1$, then $s(r) \leq 3$ as otherwise, one of the subtrees of $r$ has $m \geq 2$ nodes and $m>s(l)$ implies $p$ is not a COST. Since $s(r) \leq 3, \frac{1}{2}(s(r)-1) \leq s(l)$ and $\frac{1}{2}(s(l)-1) \leq s(r)$. So, $p$ is $\frac{1}{2}$-balanced. The same proof applies when $s(r)=1$. When $s(l)>1$ and $s(r)>1$, let $a$ and $b$ be the roots of the left and right subtrees of $l$. Since $p$ is a COST, $s(a) \leq s(r)$ and $s(b) \leq s(r)$. So, $s(l)=s(a)+s(b)+1 \leq 2 s(r)+1$ and $\frac{1}{2}(s(l)-1) \leq s(r)$. Similarly, $\frac{1}{2}(s(r)-1) \leq s(l)$. So, $\frac{1}{2}-(l, r)$. Since this proof applies to every nodes in $T$, the children of every $p$ are $\frac{1}{2}$-balanced and $T$ is $\frac{1}{2}$-balanced.

Remark There are $\frac{1}{2}$-balanced trees that are not COSTs.

While a COST is in $\mathrm{WB}(1 / 3)$ and $\mathrm{WB}(\alpha)$ trees can be maintained efficiently only for $2 / 11<\alpha \leq 1-1 / \sqrt{2} \approx 0.293$, a COST is better balanced than $\mathrm{WB}(\alpha)$ trees with $\alpha$ in the usable range. Unfortunately, we are unable to develop $\mathrm{O}(\log n)$ insert/delete algorithms for a COST.

In the next section, we develop insert and delete algorithms for $\beta$-balanced binary search trees $(\beta$-BBST) for $0<\beta \leq \sqrt{2}-1$. Note that every $(\sqrt{2}-1)$-BBST is in $\mathrm{WB}(\alpha)$ for $\alpha=1-1 / \sqrt{2}$ which is the largest permissible $\alpha$. Since our insert and delete algorithms perform rotations along the search path whenever these result in improved search cost, BBSTs are expected to have better search performance than $\mathrm{WB}(\alpha)$ trees (for $\alpha=\beta /(1+\beta)$ ). 
Each node of a $\beta$-BBST has the fields LeftChild, Size, Data, and RightChild. Since every $\beta$-BBST, $\beta>0$, is in $\mathrm{WB}(\alpha)$, for $\alpha>0, \beta$-BBSTs have height that is logarithmic in $n$, the number of nodes (provided $\beta>0$ ).

\section{Search, Insert, and Delete in a $\beta$-BBST}

To reduce notational clutter, in the rest of the paper, we abbreviate $s(a)$ by $a$ (i.e., the node name denotes subtree size).

\subsection{Search}

This is done exactly as in any binary search tree. Its complexity is $\mathrm{O}(h)$ where $h$ is the height of the tree. Notice that since each node has a size field, it is easy to perform a search based on index (i.e., find the 10'th smallest key). Similarly, our insert and delete algorithms can be adapted to indexed insert and delete.

\subsection{Insertion}

To insert a new element $x$ into a $\beta$-BBST, we first search for $x$ in the $\beta$-BBST. This search is unsuccessful (as $x$ is not in the tree) and terminates by falling off the tree. A new node $y$ containing $x$ is inserted at the point where the search falls off the tree. Let $p^{\prime}$ be the parent (if any) of the newly inserted node. We now retrace the path from $p^{\prime}$ to the root performing rebalancing rotations.

There are four kinds of rotations LL, LR, RL, and RR. LL and RR rotations are symmetric and so also are LR and RL rotations. The typical configuration before an LL rotation is performed is given in Figure 2(a). $p^{\prime}$ denotes the root of a subtree in which the insertion was made. Let $p$ be the (size of the) subtree before the insertion. Then, since the tree was a $\beta$-BBST prior to the insertion, $\beta$ - $(p, d)$. Also, for the LL rotation to be performed, we require that $(q \geq c)$ and $(q>d)$. Note that $q>d$ implies $q \geq 1$. We shall see that $\beta-(q, c)$ follows from the fact that the insertion is made into a $\beta$-BBST and from properties of the rotation. Following an LL rotation, $p^{\prime}$ is updated to be the node $p^{\prime \prime}$.

Lemma 5 [LL insertion lemma] If $[\beta-(p, d) \wedge \beta-(q, c) \wedge(q \geq c) \wedge(q>d)]$ for $0 \leq \beta \leq 1 / 2$ before the rotation, then $\beta-\left(q, g p^{\prime}\right)$ and $\beta-(c, d)$ after the rotation.

Proof Assume the before condition.

(a) $\beta(q-1) \leq c$ (as $\beta-(q, c))<g p^{\prime}$. Also, $\beta\left(g p^{\prime}-1\right)=\beta(c+d) \leq 2 \beta q$ (as $\beta \geq 0, q \geq c$ and $q>d) \leq q($ as $\beta \leq 1 / 2)$. So, $\beta$ - $\left(q, g p^{\prime}\right)$. 


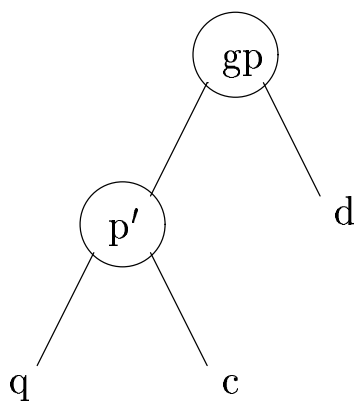

(a) before
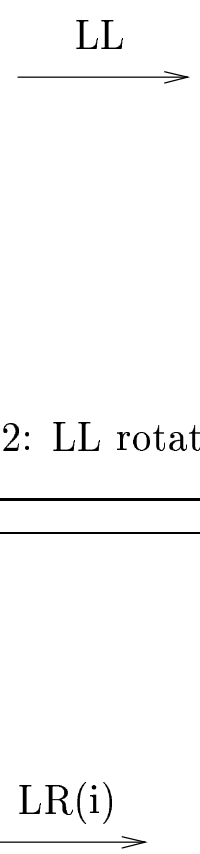

a

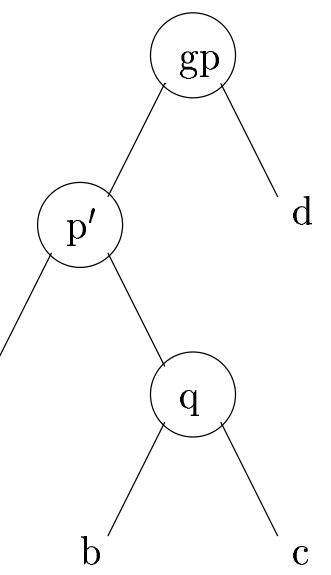

(a) before

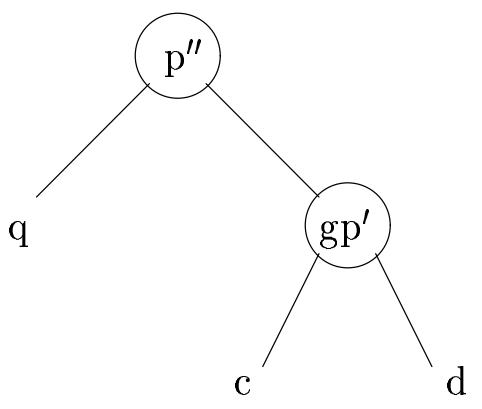

(b) after

Figure 2: LL rotation for insertion

(b) $d<q \Rightarrow d-1<q-1 \Rightarrow \beta(d-1) \leq \beta(q-1) \leq c$ (as $\beta-(q, c))$. Also, $\beta(c-1) \leq$ $\beta(q+c-1)=\beta\left(p^{\prime}-2\right)=\beta(p-1) \leq d($ as $\beta-(p, d))$. So, $\beta-(c, d)$.

In an LR rotation, the before configuration is as in Figure 2(a). However, this time $q<c$. Figure 2(a) is redrawn in Figure 3(a). In this, the node labeled $c$ in Figure 2(a) has been labeled $q$ and that labeled $q$ in Figure 2(a) has been labeled $a$. With respect to the labelings of Figure 3(a), rotation LR is applied when

$$
[(q>a) \wedge(q>d)] .
$$

The other conditions that apply when an LR rotation is performed are

$$
[\beta-(p, d) \wedge \beta-(a, q) \wedge \beta-(b, c)] .
$$


Here $p$ denotes the (size of the) left subtree of $g p$ prior to the insertion. An LR rotation is accomplished in two substeps (or two subrotations). The first of these is shown in Figure 3(b). Following an LR rotation, $p^{\prime}$ is updated to be node $q^{\prime}$.

Lemma 6 [LR substep $(i)$ insertion lemma] If $[\beta-(p, d) \wedge \beta-(a, q) \wedge \beta-(b, c) \wedge(q>a) \wedge(q>d)]$ for $0 \leq \beta \leq 1 / 2$ before the subrotation, then $\left[\beta-\left(p^{\prime \prime}, g p^{\prime}\right) \wedge\left\{\left(\beta-(a, b) \wedge \frac{\beta}{1+\beta}-(c, d)\right) \vee\left(\frac{\beta}{1+\beta}-\right.\right.\right.$ $(a, b) \wedge \beta-(c, d))\}]$ after the subrotation.

Proof Assume the before condition. First, we show that $\beta$ - $\left(p^{\prime \prime}, g p^{\prime}\right)$ after the rotation. Note that $\beta\left(p^{\prime \prime}-1\right)=\beta(a+b)=\beta(a+b+c+1)-\beta(c+1)=\beta\left(p^{\prime}-1\right)-\beta(c+1)=$ $\beta(p-1)-\beta c \leq d-\beta c \leq d<g p^{\prime}$. Also, $\beta\left(g p^{\prime}-1\right)=\beta(c+d) \leq b+\beta+\beta d(\operatorname{as} \beta-(b, c))$ $\leq b+\beta q($ as $q>d) \leq b+a+\beta($ as $\beta-(a, q))<p^{\prime \prime}$ (as $\beta \leq 1 / 2$ and $\left.p^{\prime \prime}=a+b+1\right)$. So, $\beta-\left(p^{\prime \prime}, g p^{\prime}\right)$.

Next, we prove two properties that will be used to complete the proof.

$\mathrm{P} 1: \beta(b-1) \leq a$.

To see this, note that $\beta(b-1) \leq \beta(q-1) \leq a($ as $\beta-(a, q))$.

$\mathrm{P} 2: \beta(c-1) \leq d$.

For this, observe that $p^{\prime}-1=a+q \geq \beta(q-1)+q($ as $\beta-(a, q))=(\beta+1)(q-1)+1$. So, $q-1 \leq \frac{p^{\prime}-2}{\beta+1}=\frac{p-1}{\beta+1}$. Similarly, $q-1=b+c \geq \beta(c-1)+c(\operatorname{as} \beta-(b, c))=(\beta+1)(c-1)+1$. So, $\beta(c-1) \leq \frac{\beta}{\beta+1}(q-2) \leq \frac{\beta}{\beta+1}(q-1) \leq \frac{\beta(p-1)}{(\beta+1)^{2}} \leq \frac{d}{(\beta+1)^{2}}($ as $\beta-(p, d)) \leq d$.

To complete the proof of the lemma, we need to show

$$
\left\{\left(\beta-(a, b) \wedge \frac{\beta}{1+\beta}-(c, d)\right) \vee\left(\frac{\beta}{1+\beta}-(a, b) \wedge \beta-(c, d)\right)\right\} .
$$

We do this by considering the two cases $b \geq c$ and $b<c$.

Case $b \geq c$ : Since $a<q=b+c+1, \beta(a-1) \leq \beta(b+c) \leq 2 \beta b \leq b$. This and P1 imply $\beta$ - $(a, b)$. Also, $d<q=b+c+1$. So, $\frac{\beta}{\beta+1}(d-1) \leq \frac{\beta}{\beta+1}(b+c-1)=\frac{\beta}{\beta+1} c+\frac{\beta}{\beta+1}(b-1) \leq \frac{\beta}{\beta+1} c+\frac{c}{\beta+1}$ $($ as $\beta-(b, c))=c$. This, together with P2 implies $\frac{\beta}{1+\beta}-(c, d)$. So, $\beta-(a, b) \wedge \frac{\beta}{1+\beta}-(c, d)$.

Case $b<c$ : Since $a<q=b+c+1, a-1<b+c$. So, $a-1 \leq b+c-1$ or $\frac{\beta(a-1)}{1+\beta} \leq \frac{\beta b}{1+\beta}+\frac{\beta(c-1)}{1+\beta} \leq \frac{\beta b}{1+\beta}+\frac{b}{1+\beta}($ as $\beta-(b, c))=b$. This and P1 imply $\frac{\beta}{1+\beta}-(a, b)$. Also, $d-1 \leq q-2=b+c-1$. So, $\beta(d-1) \leq \beta(b+c-1) \leq \beta(2 c-1) \leq c$. This, together with P2 implies $\beta$ - $(c, d)$. So, $\frac{\beta}{1+\beta}-(a, b) \wedge \beta-(c, d)$.

Since an LR(i) rotation can cause the tree to lose its $\beta$-balance property, it is necessary to follow this with another rotation that restores the $\beta$-balance property. It suffices to consider the two cases of Figures 4 and 5 for this follow up rotation. The remaining cases are symmetric to these. In Figures 4 and $5, p$ and $d$ denote the nodes that do not satisfy $\beta-(p, d)$. Note, however, that these nodes do satisfy $\frac{\beta}{1+\beta}-(p, d)$. 


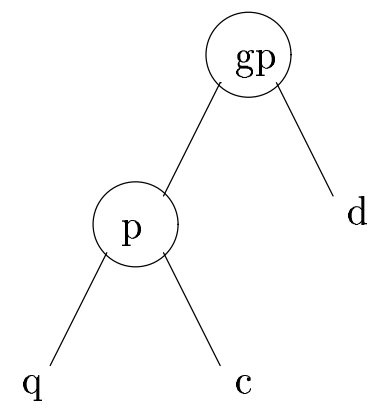

(a) before

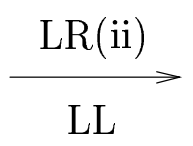

Figure 4: Case LL for LR(ii) rotation

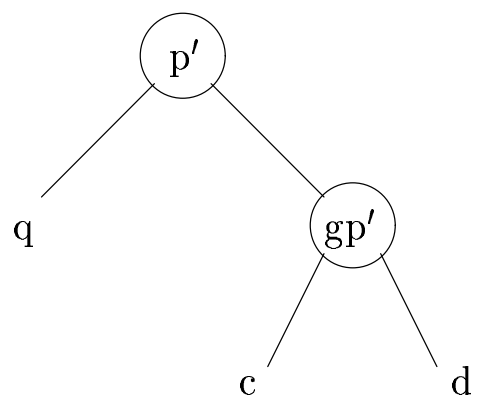

(b) after

Since the follow up rotation to LR(i) is done only when

$$
\frac{\beta}{1+\beta}-(p, d) \wedge(\neg \beta-(p, d)),
$$

either $\beta(p-1)>d$ or $\beta(d-1)>p$. When $\beta(p-1)>d$, the second substep rotation is one of the two given in Figures 4 and 5 . When $\beta(d-1)>p$, rotations symmetric to these are performed. In the following, we assume $\beta(p-1)>d$. Further, we may assume $d>0$, as $d=0$ and $\frac{\beta}{1+\beta}-(p, d)$ imply $p \leq 1$. Hence, $\beta-(p, d)$. Also, $d>0$ and $\beta(p-1)>d$ imply $p>1$.

The LR(ii) LL rotation is done when the condition

$$
\begin{gathered}
A=(q>d) \wedge(c<(1+\beta) q+(1-\beta)) \wedge B \text { where } \\
B=\frac{\beta}{1+\beta}-(p, d) \wedge(\neg \beta-(p, d)) \wedge \beta-(q, c) \wedge(\beta(p-1)>d>0) .
\end{gathered}
$$

Lemma 7 [Case LR(ii) LL rotation] If $A$ holds before the rotation of Figure 4, then $\beta$ $\left(q, g p^{\prime}\right)$ and $\beta-(c, d)$ after the rotation provided $0<\beta \leq \sqrt{2}-1$.

Proof (a) $\beta-\left(q, g p^{\prime}\right)$ :

$\beta(q-1) \leq c(\operatorname{as} \beta-(q, c))<g p^{\prime}$. Also, $\beta\left(g p^{\prime}-1\right)=\beta(c+d)<\beta((1+\beta) q+(1-\beta)+d) \leq$ $\beta(1+\beta) q+\beta(1-\beta)+\beta(q-1)($ as $q>d)=\beta(2+\beta) q-\beta^{2}<q($ as $\beta(2+\beta) \leq 1$ for $0<\beta \leq \sqrt{2}-1)$. So, $\beta-\left(q, g p^{\prime}\right)$.

(b) $\beta-(c, d)$ :

$\beta(d-1)<\beta(q-1) \leq c($ as $\beta-(q, c))$. And, $\beta(c-1)=\frac{\beta^{2}}{1+\beta}(c-1)+\frac{\beta}{1+\beta}(c-1) \leq$ $\frac{\beta}{1+\beta} q+\frac{\beta}{1+\beta}(c-1)=\frac{\beta}{1+\beta}(q+c-1)=\frac{\beta}{1+\beta}(p-2)<\frac{\beta}{1+\beta}(p-1) \leq d\left(\right.$ as $\left.\frac{\beta}{1+\beta}-(p, d)\right)$. So, $\beta-(c, d)$. 


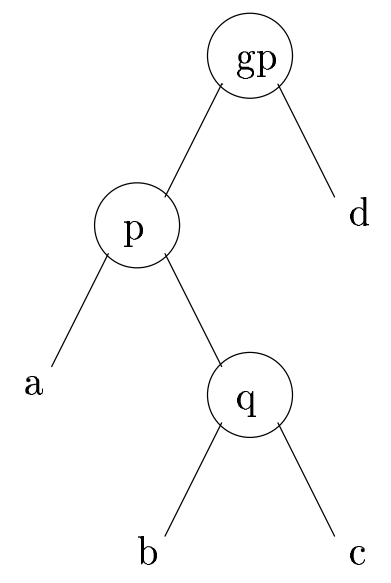

(a) before

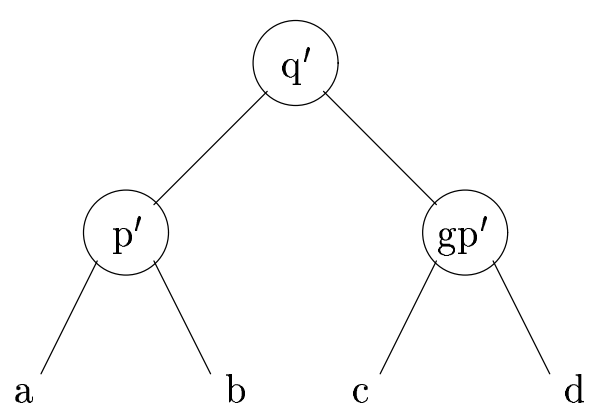

(b) after

Figure 5: Case LR for LR(ii) rotation

Lemma 8 If $(c<(1+\beta) q+(1-\beta)) \wedge(\beta(p-1)>d)$ in Figure 4, then $d \leq q$ provided $0<\beta \leq \sqrt{2}-1$.

Proof Since $d<\beta(p-1)=\beta(q+c)<\beta(q+(1+\beta) q+1-\beta)=\beta(\beta+2) q+\beta(1-\beta)<q+1$ $($ as $\beta(\beta+2) \leq 1$ and $\beta(1-\beta)<1$ for $0<\beta \leq \sqrt{2}-1)$. So, $d \leq q$.

So, the only time an $\mathrm{LR}$ (ii) LL rotation is not done is when $C=\left(C_{1} \vee C_{2}\right) \wedge B$ holds where $C_{1}=(q=d) \wedge(c<(1+\beta) q+1-\beta)$ and $C_{2}=c \geq(1+\beta) q+(1-\beta)$. At this time, the LR rotation of Figure 5 is done. In terms of the notation of Figure 5 , the condition $C$ becomes $D=\left(D_{1} \vee D_{2}\right) \wedge E$ where $D_{1}=(a=d) \wedge(q<(1+\beta) a+1-\beta), D_{2}=q \geq(1+\beta) a+1-\beta$, and

$$
E=\frac{\beta}{1+\beta}-(p, d) \wedge \neg \beta-(p, d) \wedge \beta-(a, q) \wedge \beta-(b, c) \wedge(\beta(p-1)>d>0) .
$$

Lemma 9 When an LR(ii) LR rotation is performed and $\beta \leq \sqrt{2}-1, q>d$ and so search cost is reduced.

Proof If $D_{1}$, then since $d<\beta(p-1)=\beta(a+q)=\beta(d+q), q>d / \beta-d>d$ as $\beta \leq \sqrt{2}-1$. If $D_{2}$, then $d<\beta(p-1)=\beta(a+q) \leq \beta\left(\frac{q-1+\beta}{1+\beta}+q\right)=\frac{\beta(2+\beta)}{1+\beta} q-\frac{\beta(1-\beta)}{1+\beta}<\frac{\beta(2+\beta)}{1+\beta} q \leq q$ (as $\beta \leq \sqrt{2}-1)$.

Lemma 10 When $(d=a) \wedge \beta-(b, c) \wedge(\beta(p-1)>d) \wedge(\beta \leq \sqrt{2}-1)$ (see Figure 5), $\beta(a-1) \leq b$ and $\beta(d-1) \leq c$. 
Proof Since $\beta(p-1)>d$ and $d=a, \beta(p-1)>a$ or $\beta(a+q)>a$ or $a(1-\beta)<\beta q$ or $a<\frac{\beta}{1-\beta} q$. So, $\beta(a-1)<\frac{\beta^{2}}{1-\beta} q-\beta=\frac{\beta^{2}}{1-\beta}(b+c+1)-\beta$.

If $c \leq \frac{b}{\beta}+\beta$, then

$$
\begin{aligned}
\beta(a-1) & <\frac{\beta^{2}}{1-\beta}\left(b+\frac{b}{\beta}+\beta+1\right)-\beta \\
& =\frac{\beta(\beta+1) b}{1-\beta}+\frac{\beta^{2}(\beta+1)}{1-\beta}-\beta \\
& =\frac{\beta(\beta+1) b}{1-\beta}+\frac{\beta\left(\beta^{2}+\beta-1+\beta\right)}{1-\beta} \\
& =\frac{\beta(\beta+1) b}{1-\beta}+\frac{\beta\left(\beta^{2}+2 \beta-1\right)}{1-\beta} \\
& \left.\leq b \text { (as } \beta(\beta+1) \leq 1-\beta \text { for } \beta \leq \sqrt{2}-1 \text { and } \beta^{2}+2 \beta-1 \leq 0 \text { for } \beta \leq \sqrt{2}-1\right) .
\end{aligned}
$$

Since $\beta(c-1) \leq b, c \leq \frac{b}{\beta}+1$. So,

$$
\beta(a-1)<\frac{\beta^{2}}{1-\beta}(b+c+1) \leq \frac{\beta^{2}}{1-\beta}\left(b+\frac{b}{\beta}+2\right) \leq \frac{\beta(\beta+1) b}{1-\beta}+\frac{3 \beta^{2}-\beta}{1-\beta} .
$$

So,

$$
a-1<\frac{\beta+1}{1-\beta} b+\frac{3 \beta-1}{1-\beta} .
$$

However, since $\beta^{2}+2 \beta-1 \leq 0$ for $\beta \leq \sqrt{2}-1,(1+\beta) /(1-\beta) \leq \frac{1}{\beta}$ and $(3 \beta-1) /(1-\beta) \leq \beta$. So, $a-1<b / \beta+\beta$. If $a \geq c+1$, then $c \leq a-1<b / \beta+\beta$. We have already shown that for $c \leq b / \beta+\beta, \beta(a-1) \leq b$. So, assume $a<c+1$. Now, $a \leq c$ and $\beta(a-1) \leq \beta(c-1) \leq b$ (as $\beta-(b, c))$. So, $\beta(a-1) \leq b$ in all cases. $\beta(a-1) \leq c$ may be shown in a similar way. Since $a=d$, we get $\beta(d-1) \leq c$.

Lemma 11 [Case LR(ii) LR rotation] If $D$ holds before the rotation of Figure 5, then $\beta$ $\left(p^{\prime}, g p^{\prime}\right), \beta-(a, b)$, and $\beta-(c, d)$ following the rotation provided $0<\beta \leq \sqrt{2}-1$.

Proof (a) $\beta-\left(p^{\prime}, g p^{\prime}\right)$ :

$\beta\left(g p^{\prime}-1\right)=\beta(c+d) \leq b+\beta+\beta d($ as $\beta-(b, c)) \leq b+\beta+\beta q($ from Lemmas 9 and $10, q \geq d)$ $\leq b+\beta+a+\beta=a+b+2 \beta<a+b+1=p^{\prime}$. Also, since $\frac{\beta}{1+\beta}-(p, d)$ and $q \geq d, \beta(p-1) \leq(\beta+1) d$ or $\beta(a+q) \leq(\beta+1) d$ or $a+q \leq\left(1+\frac{1}{\beta}\right) d$ or $a \leq\left(1+\frac{1}{\beta}\right) d-q \leq\left(1+\frac{1}{\beta}\right) d-d=d / \beta$. So, $\beta\left(p^{\prime}-1\right)=\beta(a+b)<d+\beta b \leq d+c+\beta($ as $\beta-(b, c))<d+c+1=g p^{\prime}$.

(b) $\beta-(a, b)$ :

Since $b \leq q$ and $\beta-(a, q), \beta(b-1) \leq \beta(q-1) \leq a$. 
When $D_{1}, \beta(a-1) \leq b$ was proved in Lemma 10. So, $\beta-(a, b)$.

When $D_{2}, q \geq a(1+\beta)+1-\beta$. So,

$$
a \leq \frac{q}{1+\beta}-\frac{1-\beta}{1+\beta}=\frac{b+c+1}{1+\beta}-\frac{1-\beta}{1+\beta} .
$$

So,

$$
\beta(a-1) \leq \frac{\beta b+\beta c+\beta}{1+\beta}-\frac{1-\beta}{1+\beta} \beta-\beta \leq \frac{\beta b+b+2 \beta}{1+\beta}-\frac{1-\beta}{1+\beta} \beta-\beta=b .
$$

So, $\beta-(a, b)$.

(c) $\beta-(c, d)$ :

Note that $\beta(c-1)<\beta(q-1)<\frac{\beta}{1+\beta}(q-1)<\frac{\beta}{1+\beta}(p-1) \leq d$.

When $D_{1}, \beta(d-1) \leq c$ was proved in Lemma 10. So, $\beta-(c, d)$.

When $D_{2}$, if $d<b+1$, then $d \leq b$ and $\beta(d-1) \leq \beta(b-1) \leq c$. So, assume $d \geq b+1$. Now, $b \leq d-1<\beta(p-1)-1$. So,

$$
\begin{aligned}
b & <\beta(a+b+c+1)-1 \\
& \leq \beta\left(\frac{q-1+\beta}{1+\beta}+b+c+1\right)-1 \\
& =\frac{\beta}{1+\beta}(b+c+\beta+(1+\beta)(b+c+1))-1 \\
& \leq \frac{\beta}{1+\beta}\left(\frac{c}{\beta}+1+c+\beta+(1+\beta)\left(\frac{c}{\beta}+1+c+1\right)\right)-1 \\
& =\frac{\beta}{1+\beta}\left(\frac{\beta+\beta}{\beta} c+(1+\beta)+(1+\beta)\left(\frac{1+\beta}{\beta} c+2\right)\right)-1 \\
& =c+\beta+(1+\beta) c+2 \beta-1 \\
& =(2+\beta) c+3 \beta-1<(2+\beta) c+\beta(\operatorname{as} \beta \leq \sqrt{2}-1) \\
& \leq \frac{c}{\beta}+\beta(\operatorname{as} \beta \leq \sqrt{2}-1) .
\end{aligned}
$$

Also, from $d<\beta(p-1)$ and the above derivation, we get

$$
\begin{aligned}
d & <\frac{\beta}{1+\beta}(b+c+\beta+(1+\beta)(b+c+1)) \\
& \leq \frac{\beta}{1+\beta}\left(\frac{c}{\beta}+\beta+c+\beta+(1+\beta)\left(\frac{c}{\beta}+\beta+c+1\right)\right) \\
& =\frac{\beta}{1+\beta}\left(\frac{\beta+1}{\beta} c\right)+\frac{2 \beta^{2}}{1+\beta}+\beta\left(\frac{1+\beta}{\beta} c\right)+\beta(\beta+1) \\
& =(2+\beta) c+\frac{2 \beta^{2}}{1+\beta}+\beta(\beta+1) \\
& =(2+\beta) c+\frac{2 \beta^{2}+\beta^{2}+\beta^{3}+\beta+\beta^{2}}{1+\beta}
\end{aligned}
$$




$$
\begin{aligned}
& =(2+\beta) c+\frac{\beta^{3}+4 \beta^{2}+\beta}{1+\beta} \\
& \leq(2+\beta) c+1\left(\text { as } \beta^{3}+4 \beta^{2}+\beta<1+\beta \text { for } \beta \leq \sqrt{2}-1\right) .
\end{aligned}
$$

So, $\beta(d-1) \leq \beta(2+\beta) c \leq c$ (as $\beta \leq \sqrt{2}-1)$. So, $\beta-(c, d)$.

Theorem 2 If $T$ is $\beta$-balanced, $0 \leq \beta \leq \sqrt{2}-1$, prior to insertion, it is so following the insertion.

Proof Follows from Lemmas 5 through 11.

Lemma 12 The time needed to do an insertion in an $n$ node $\beta-B B S T$ is $O(\log n)$ provided $0<\beta \leq \sqrt{2}-1$.

Proof Follows from the fact that insertion takes $\mathrm{O}(h)$ time where $h$ is the tree height and $h=\mathrm{O}(\log n)$ when $\beta>0$ (Lemmas 1 and 3$)$.

\subsection{Deletion}

To delete element $x$ from a $\beta$-BBST, we first use the unbalanced binary search tree deletion algorithm of [SAHN98] to delete $x$ and then perform a series of rebalancing rotations. The steps are:

Step 1 [Locate $x$ ] Search the $\beta$-BBST for the node $y$ that contains $x$. If there is no such node, terminate.

Step 2 [Delete $x$ ] If $y$ is a leaf, set $d^{\prime}$ to nil, $g p$ to the parent of $y$, and delete node $y$. If $y$ has exactly one child, set $d^{\prime}$ to be this child; change the pointer from the parent (if any) of $y$ to point to the child of $y$; delete node $y$; set $g p$ to be the parent of $d^{\prime}$. If $y$ has two children, find the node $z$ in the left subtree of $y$ that has largest value; move this value into node $y$; set $y=z$; go to the start of Step 2. \{ note that the new $y$ has either 0 or 1 child \}

Step 3 [Rebalance] Retrace the path from $d^{\prime}$ to the root performing rebalancing rotations.

There are four rebalancing rotations LL, LR, RR, and RL. Since LL and RR as well as LR and RL are symmetric rotations, we describe LL and LR only. The discussion is very 


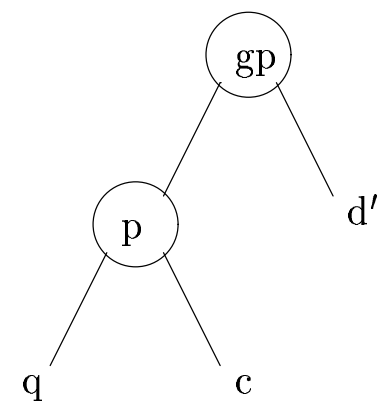

(a) before

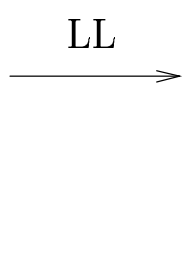

Figure 6: LL rotation for deletion

similar to the case of insertion. The differences in proofs are due to the fact that a deletion reduces the size of encountered subtrees by 1 while an insertion increases it by 1 . In an LL rotation, the configuration just before and after the rotation is shown in Figure 6. This rotation is performed when $q \geq c$ and $q>d^{\prime}$. Following the rotation, $d^{\prime}$ is updated to the node $p^{\prime}$.

Let $d$ denote the size of the right subtree of $g p$ before the deletion. So, $d=d^{\prime}+1$. Since prior to the deletion the $\beta$-BBST was $\beta$-balanced, it follows that $\beta-(p, d)$ and $\beta-(q, c)$.

Lemma 13 [LL deletion lemma] If $[\beta-(p, d) \wedge \beta-(q, c) \wedge(q \geq c) \wedge(q>d) \wedge(1 / 3 \leq \beta \leq 1 / 2)]$ before the rotation, then $\left[\beta-\left(q, g p^{\prime}\right) \wedge \beta-\left(c, d^{\prime}\right)\right]$ after the rotation.

Proof Similar to Lemma 5.

In an LR rotation, the before configuration is as in Figure 6(a). However, this time $q<c$. Figure 6(a) is redrawn in Figure 7(a). In this, the node labeled $c$ in Figure 6(a) has been relabeled $q$ and that labeled $q$ in Figure 6(a) has been relabeled $a$. With respect to the labelings of Figure 7(a), rotation LR is applied when $\left[(q>a) \wedge\left(q>d^{\prime}\right)\right]$. The other conditions that apply when an LR rotation is performed are $[\beta-(p, d) \wedge \beta-(a, q) \wedge \beta-(b, c)]$. Here $d$ denotes the (size of) right subtree of $g p$ prior to the deletion. As in the case of insertion, an LR rotation is accomplished in two substeps (or two subrotations). The first of these is shown in Figure 7. Following an LR rotation, $d^{\prime}$ is updated to node $q^{\prime}$.

Lemma 14 [LR substep (i) deletion lemma] If $\left[\beta-(p, d) \wedge \beta-(a, q) \wedge \beta-(b, c) \wedge(q>a) \wedge\left(q>d^{\prime}\right)\right]$ before the subrotation $L R(i)$, then $\left[\beta-\left(p^{\prime}, g p^{\prime}\right) \wedge\left\{\left(\beta-(a, b) \wedge \frac{\beta}{1+\beta}-\left(c, d^{\prime}\right)\right) \vee\left(\frac{\beta}{1+\beta}-(a, b) \wedge \beta-\left(c, d^{\prime}\right)\right)\right\}\right]$ after the subrotation provided $1 / 3 \leq \beta \leq 1 / 2$. 


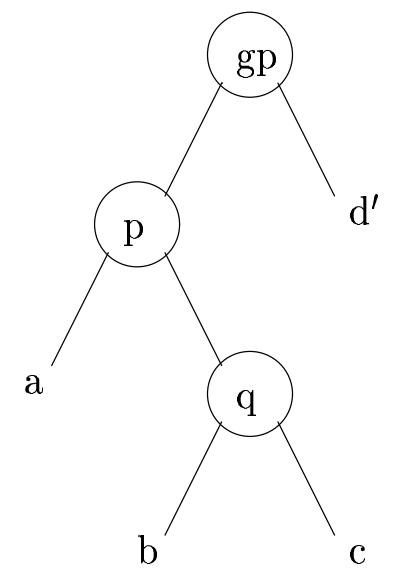

(a) before

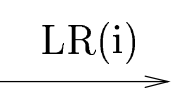

(b) after substep (i)

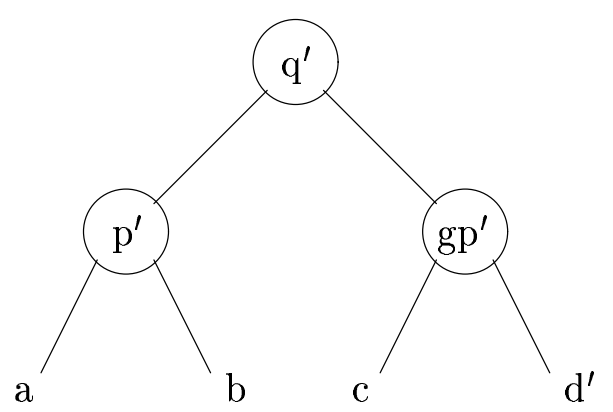

Figure 7: LR rotation for deletion

Proof Assume the before condition.

(a) If $b=c=0$, then $q=b+c+1=1$. Furthermore, $(q>a)$ and $\left(q>d^{\prime}\right)$ imply $a=d^{\prime}=0$. So, $g p^{\prime}=p^{\prime}=1$. Hence, $\left[\frac{1}{2}-\left(p^{\prime}, g p^{\prime}\right) \wedge \frac{1}{2}-(a, b) \wedge \frac{1}{2}-\left(c, d^{\prime}\right)\right]$

(b) If $b=1$ and $c=0$, then $q=2, a \leq 1$, and $d^{\prime} \leq 1$. So, $1 \leq p^{\prime} \leq 3$ and $1 \leq g p^{\prime} \leq 2$. Hence, $\left[\frac{1}{2}-\left(p^{\prime}, g p^{\prime}\right) \wedge \frac{1}{2}-(a, b) \wedge \frac{1}{2}-\left(c, d^{\prime}\right)\right]$

(c) If $b=0$ and $c=1$, then $q=2, a \leq 1$, and $d^{\prime} \leq 1$. So, $1 \leq p^{\prime} \leq 2$ and $1 \leq g p^{\prime} \leq 3$. Hence, $\left[\frac{1}{2}-\left(p^{\prime}, g p^{\prime}\right) \wedge \frac{1}{2}-(a, b) \wedge \frac{1}{2}-\left(c, d^{\prime}\right)\right]$

As a result of $(\mathrm{a})-(\mathrm{c})$, to complete the proof, we may assume that $b \geq 1$ and $c \geq 1$. So, $q \geq 3, a \geq 1($ as $\beta-(a, q) \Rightarrow \beta(q-1) \leq a$ or $a \geq 2 \beta>0), p=a+q+1 \geq 5, d \geq 2$ (as $\beta-(p, d) \Rightarrow \beta(p-1) \leq d$ and $\beta \geq 1 / 3)$, and $d^{\prime}=d-1 \geq 1$.

First, we show that $\beta$ - $\left(p^{\prime}, g p^{\prime}\right)$. For this, note that $a+b+c+1=p-1$. From $\beta-(p, d)$, it follows that $\beta(a+b+c+1)=\beta(p-1) \leq d$. So, $\beta(a+b) \leq d-\beta c-\beta$. From Figure $7(\mathrm{~b})$, we see that $\beta\left(p^{\prime}-1\right)=\beta(a+b)$. Hence, $\beta\left(p^{\prime}-1\right) \leq d-\beta c-\beta=d^{\prime}-\beta c+1-\beta \leq d^{\prime}+1-2 \beta<g p^{\prime}$. Also, $\beta\left(g p^{\prime}-1\right)=\beta\left(c+d^{\prime}\right) \leq b+\beta+\beta d^{\prime}(\operatorname{as} \beta-(b, c))<b+\beta q+\beta\left(\right.$ as $\left.q>d^{\prime}\right) \leq b+a+2 \beta(\operatorname{as} \beta-(a, q))$ $<p^{\prime}$. So, $\beta$ - $\left(p^{\prime}, g p^{\prime}\right)$.

Next, we prove two properties that will be used to complete the proof.

$\mathrm{P} 1: \beta(b-1) \leq a$.

To see this, note that $\beta(b-1)<\beta(q-1) \leq a(\operatorname{as} \beta-(a, q))$.

$\mathrm{P} 2: \beta(c-1) \leq d^{\prime}$.

For this, observe that $\beta(c-1) \leq \beta(q-2)$ (as $c \leq q-1) \leq \beta(p-4)$ (as $q=p-a-1$ and $a \geq 1)=\beta(p-1)-3 \beta \leq d-1($ as $\beta-(p, d)$ and $\beta \geq 1 / 3)=d^{\prime}$. 
To complete the proof of the lemma, we need to show

$$
\left\{\left(\beta-(a, b) \wedge \frac{\beta}{1+\beta}-\left(c, d^{\prime}\right)\right) \vee\left(\frac{\beta}{1+\beta}-(a, b) \wedge \beta-\left(c, d^{\prime}\right)\right)\right\}
$$

For this, consider the two cases $b \geq c$ and $b<c$ (as in Lemma 6).

Case $b \geq c$ : Since $a<q=b+c+1, \beta(a-1)<\beta(b+c) \leq 2 \beta b \leq b$. This, together with P1 implies $\beta$ - $(a, b)$. Also, $d^{\prime}<q=b+c+1$. So, $\frac{\beta}{\beta+1}\left(d^{\prime}-1\right) \leq \frac{\beta}{\beta+1}(b+c-1)=\frac{\beta}{\beta+1} c+\frac{\beta}{\beta+1}(b-1) \leq$ $\frac{\beta}{\beta+1} c+\frac{c}{\beta+1}=c$. This, together with P2 implies $\frac{\beta}{1+\beta}-\left(c, d^{\prime}\right)$. So, $\beta-(a, b) \wedge \frac{\beta}{1+\beta}-\left(c, d^{\prime}\right)$.

Case $b<c$ : Since $a<q=b+c+1, a-1<b+c$. So, $a-1 \leq b+c-1$ or $\frac{\beta(a-1)}{1+\beta} \leq$ $\frac{\beta b}{1+\beta}+\frac{\beta(c-1)}{1+\beta} \leq \frac{\beta b}{1+\beta}+\frac{b}{1+\beta}=b$. This and P1 imply $\frac{\beta}{1+\beta}-(a, b)$. Also, $d^{\prime}-1 \leq q-2=b+c-1$. So, $\beta\left(d^{\prime}-1\right) \leq \beta(b+c-1)<\beta(2 c-1)<c$. This and P2 imply $\beta$ - $\left(c, d^{\prime}\right)$. Hence, $\frac{\beta}{1+\beta}$ $(a, b) \wedge \beta-\left(c, d^{\prime}\right)$.

The substep(ii) rotations are the same as for insertion.

Theorem 3 If $T$ is $\beta$-balanced, then following a deletion the resulting tree $T^{\prime}$ is also $\beta$ balanced provided $1 / 3 \leq \beta \leq \sqrt{2}-1$.

When $0<\beta<1 / 3$, we need to augment the LL rotation by a transformation for the case $d^{\prime}=0$. When $d^{\prime}=0, \beta(p-1) \leq d=d^{\prime}+1=1$. So, $p \leq 1 / \beta+1$ and $g p=p+d^{\prime}+1 \leq 1 / \beta+2$. To $\beta$-balance at $g p$, the at most $1 / \beta+2$ nodes in $g p$ are rearranged into any $\beta$-BBST in constant time (as $1 / \beta+2$ is a constant). When $d^{\prime}>0$, the proof of Lemma 13 part (b) can be changed to show $\beta(c-1) \leq d^{\prime}$ for $0<\beta \leq \sqrt{2}-1$. The new proof is: since $c \leq q, c \leq(p-1) / 2$ and $\beta(c-1) \leq \beta(p-1) / 2-\beta \leq d / 2-\beta=d-d / 2-\beta \leq d-1-\beta<d^{\prime}$. The LR rotation needs to be augmented by a transformation for the case $d^{\prime}=d-1<\frac{1}{\beta(2+\beta)}-1$. At this time, $\beta(p-1) \leq d<\frac{1}{\beta(2+\beta)}$. So, $g p=p+d<\frac{1}{\beta^{2}(2+\beta)}+1+\frac{1}{\beta(2+\beta)}$. To $\beta$-balance at $g p$, we rearrange the fewer than $\frac{1}{\beta^{2}(2+\beta)}+1+\frac{1}{\beta(2+\beta)}$ nodes in the subtree, in constant time, into any $\beta$-balanced tree. When $d^{\prime} \geq \frac{1}{\beta(2+\beta)}-1$, the proof for $\beta(c-1) \leq d^{\prime}$ in Lemma 14 needs to be changed to show that the LR substep(i) lemma holds. The new proof is:

$$
\begin{aligned}
d & \geq \beta(p-1)=\beta(a+b+c+1) \geq \beta(\beta(q-1)+b+c+1) \\
& =\beta(\beta(b+c)+b+c+1) \\
& \geq \beta((1+\beta) \beta(c-1)+(1+\beta) c+1) \\
& =\beta\left((1+\beta)^{2}(c-1)+2+\beta\right) .
\end{aligned}
$$

So, $\beta(c-1) \leq \frac{d-2 \beta-\beta^{2}}{(1+\beta)^{2}} \leq d-1\left(\right.$ as $\left.d \geq \frac{1}{\beta(2+\beta)}\right)=d^{\prime}$.

Also, note that when $\beta=0$, all trees are $\beta$-balanced so the rotations (while not needed) preserve balance. 
Theorem 4 With the special handling of the case $d^{\prime}=0$, the tree $T^{\prime}$ resulting from a deletion in a $\beta$-BBST is also $\beta$-balanced for $0 \leq \beta \leq \sqrt{2}-1$.

Lemma 15 The time needed to delete an element from an $n$ node $\beta-B B S T$ is $O(\log n)$ provided $0<\beta \leq \sqrt{2}-1$.

\subsection{Enhancements}

Since our objective is to create search trees with minimum search cost, the rebalancing rotations may be performed at each positioning of $g p$ during the backward restructuring pass so long as the conditions for the rotation apply rather than only at $g p$ positions where the tree is unbalanced.

Consider Figure 2(a). If $p^{\prime}<d$, then the conditions of Lemmas 5 and 6 cannot apply as $q<p^{\prime}<d$. However, it is possible that $e>p^{\prime}$ where $e$ is the size of either the left or right subtree of $d$. In this case, an RR or RL rotation would reduce the total search cost. The proofs of Lemmas 5 and 6 are easily extended to show that these rotations would preserve balance even though no insertion was done in the subtree $d$. The same observation applies to deletion. Hence the backward restructuring pass for the insert and delete operations can determine the need for a rotation at each $g p$ location as below ( $l$ and $r$ are, respectively, the left and right children of $g p$ ).

if $s(l)>s(r)$ then check conditions for an LL and LR rotation

else check conditions for an RR and RL rotation.

The enhanced restructuring procedure used for insertion and deletion is given in Figure 8. In the RR and RL cases, we have used the relation ' $\geq$ ' rather than ' $>$ ' as this results in better observed run time.

Since it can be shown that the rotations preserve balance even when there has been no insert or delete, we may check the rotation conditions during a search operation and perform rotations when these improve total search cost.

Finally, we note that it is possible to use other definitions of $\beta$-balance. For example, we could require $\beta(s(a)-2)<s(b)$ and $\beta(s(b)-2)<s(a)$ for $\beta$ - $(a, b)$. One can show that the development of this paper applies to these modifications also. Furthermore, when this new definition is used, the number of comparisons in the second substep of the LR and RL rotations is reduced by one.

\subsection{Top Down Algorithms}

As in the case of red/black and $\mathrm{WB}(\alpha)$ trees, it is possible to perform, in $\mathrm{O}(\log n)$ time, inserts and deletes using a single top to bottom pass. The algorithms are similar to those 
procedure Restructuring ;

begin

while $(g p)$ do

begin

if $s($ gp.left $)>s($ gp.right $)$ then $\{$ check conditions for an LL and LR rotation $\}$ begin

$p=$ gp.left

if $(s($ p.left $)>s($ p.right $))$ then

begin if $(s($ p.left $)>s($ gp.right $))$ then do LL rotation; end else

begin

if $(s($ p.right $)>s($ gp.right $))$ then $\{\operatorname{LR}\}$

begin

do LR rotation ;

\{now notations $a, b, c$, and $d$ follow from figure $1(\mathrm{~b})\}$

if $(\beta(s(a)-1)>s(b))$ then

if $((s($ a.right $)<(1+\beta) s($ a.left $)+1-\beta)$ and

$(s(b)<s($ a.left $)))$ then

do LL rotation

else do LR rotation

else if $(\beta(s(d)-1)>s(c))$ then

if $((s($ d.left $)<(1+\beta) s($ d.right $)+1-\beta)$ and $(s(c)<s($ d.right $)))$ then

do $\mathrm{RR}$ rotation

else do RL rotation ;

end

end

end

else $\{$ check conditions for an RR and RL rotation $\}$

begin

$p=$ gp.right

if $(s($ p.left $)>s($ p.right $))$ then

begin

if $(s(p . l e f t) \geq s(g p . l e f t))$ then $\{\mathrm{RL}\}$

do symmetric to the above LR case ;

end

else

begin if $(s($ p.right $) \geq s(g p . l e f t))$ then do $\mathrm{RR}$ rotation; end ; end ;

gp $=$ gp.parent

end ;

end ;

Figure 8: Restructuring procedure 
already presented.

\section{$5 \quad$ Simple $\beta$-BBSTs}

The development of Section 4 was motivated by our desire to construct trees with minimal search cost. If instead, we desire only logarithmic performance per operation, we may simplify the restructuring pass so that rotations are performed only at nodes where the $\beta$-balance property is violated. In this case, we may dispense with the LL/RR rotations and the first substep of an LR/RL rotation. Only LR/RL substep (ii) rotations are needed. To see this, observe that Lemmas 7 and 11 show that the second substep rotations rebalance at $g p$ (see Figures 4 and 5 ) provided $\frac{\beta}{1+\beta}-(p, d)$ (The remaining conditions are ensured by the bottom-up nature of restructuring and the fact the tree was $\beta$-balanced prior to the insert or delete).

If the operation that resulted in loss of balance at $g p$ was an insert, then $\beta(p-2) \leq d$ (as $p>d$, the insert took place in subtree $p$ and $g p$ was $\beta$-balanced prior to the insert) and $\beta(p-1)>d$ (gp is not $\beta$-balanced following the insert). For the substep (ii) rotation to restore balance, we need $\beta(p-1) \leq(1+\beta) d$. This is assured if $d+\beta \leq(\beta+1) d$ (as $\beta(p-2) \leq d)$. So, we need $d \geq 1$. If $d<1$, then $d=0$. Now $\beta(p-2) \leq d$ and $\beta(p-1)>d$ imply $p=2$. One may verify that when $p=2$, the LR(ii) rotations restore balance.

If the loss of $\beta$-balance at $g p$ is the result of a deletion (say from its right subtree), then $\beta(p-1) \leq d+1$ (as $g p$ was $\beta$-balanced prior to the delete). For the substep (ii) rotation to accomplish the rebalancing, we need $\beta(p-1) \leq(\beta+1) d$. This is guaranteed if $d+1 \leq(\beta+1) d$ or $d \geq 1 / \beta$. When $d<1 / \beta$ and $\beta \geq 1 / 3, d \leq 2$. Since $\beta(p-1) \leq d+1$ and $\beta \geq 1 / 3$, when $d=2, p \leq 10$; when $d=1, p \leq 7$; and when $d=0, p \leq 4$. We may verify that for all these cases, the LR(ii) rotations restore balance. Hence, the only problematic case is when $\beta<1 / 3$ and $d<1 / \beta$.

When $\beta<1 / 3$, an LL rotation fails to restore balance only when $d=0$ (see discussion following Theorem 3). So we need to rearrange the at most $1 / \beta+2$ nodes in $g p$ into any $\beta$-balanced tree when $d=0$. An LR rotation fails only when $d<\frac{1}{\beta(2+\beta)}-1$. To see this, note that in the terminology of Lemma $14, d$ is $d^{\prime}$. The proof of P2 is extended to the case $\beta \leq 1 / 3$ when $d^{\prime} \geq \frac{1}{\beta(2+\beta)}-1$. Also, since $d^{\prime}<1 / \beta$, for the case $b \geq c$, we get $\beta\left(d^{\prime}-1\right)<1-\beta<c$ (as $c \geq 1)$. For the case $b<c$, we need to show $\beta(a-1) \leq b$. Since an LR rotation is done only when condition $D 1 \vee D 2$ holds, from Lemmas 10 and 11, it follows that $\beta(a-1) \leq b$. So, an LR rotation rebalances when $\beta<1 / 3$ provided $d \geq \frac{1}{\beta(2+\beta)}-1$. For smaller $d$, the at most $\frac{1}{\beta^{2}(2+\beta)}+\frac{1}{\beta(2+\beta)}+1$ nodes in the subtree $g p$ may be directly rearranged into a $\beta$-balanced tree.

The restructuring algorithm for simple $\beta$-BBSTs is given in Figures 9 and 10 . The 


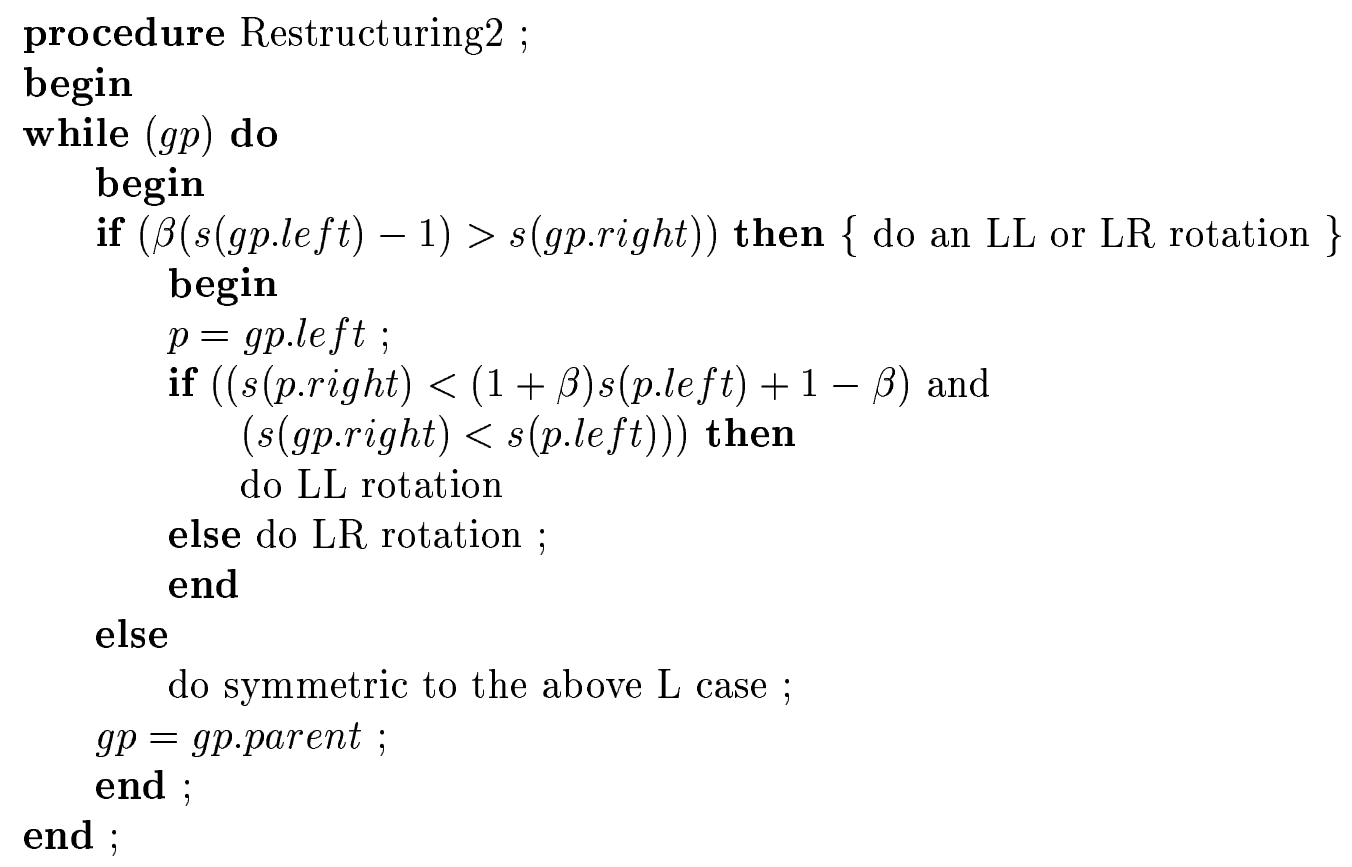

Figure 9: Simple restructuring procedure for insertion

algorithm of Figure 9 is used following an insert and that of Figure 10 after a delete.

Simple $\beta$-BBSTs are expected to have higher search cost than the $\beta$-BBSTs of Section 4. However, they are a good alternative to traditional $\mathrm{WB}(\alpha)$ trees as they are expected to be "better balanced". To see this, note that from the proof of Lemma 3, the balance, $B(p)$, at any node $p$ in a $\beta$-balanced tree satisfies

$$
\begin{aligned}
\frac{1}{B(p)} & =1+\frac{s(r)+1}{s(l)+1} \\
& \geq 1+\frac{1}{1 / \beta+\frac{2 \beta-1}{\beta(s(r)+1)}} \\
& =\frac{1+\frac{1}{\beta}+\frac{2 \beta-1}{\beta(s(r)+1)}}{\frac{1}{\beta}+\frac{2 \beta-1}{\beta(s(r)+1)}} .
\end{aligned}
$$

So,

$$
B(p) \leq 1-\frac{1}{1+\frac{1}{\beta}+\frac{2 \beta-1}{\beta(s(r)+1)}} .
$$

Also, since $s(r)-1 \leq s(l) / \beta, s(r)+1 \leq s(l) / \beta+2$. Hence, $1+\frac{s(r)+1}{s(l)+1} \leq 1+\frac{s(l)}{\beta(s(l)+1)}+\frac{2}{s(l)+1}$. So,

$$
\begin{aligned}
B(p) & \geq \frac{1}{1+\frac{1}{\beta}-\frac{1}{\beta(s(l)+1)}+\frac{2}{s(l)+1}} \\
& =\frac{1}{1+\frac{1}{\beta}+\frac{2 \beta-1}{\beta(s(l)+1)}} .
\end{aligned}
$$




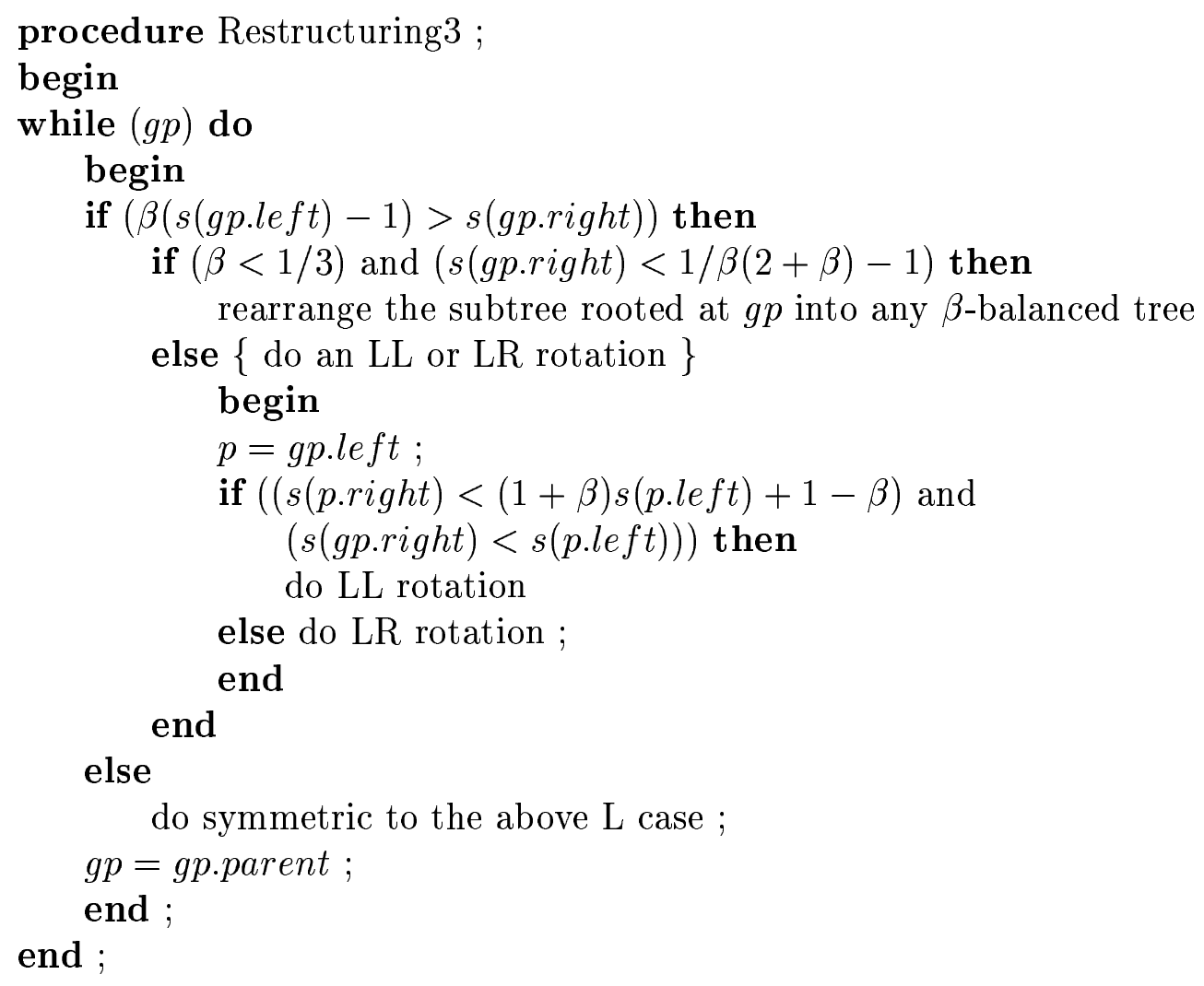

Figure 10: Simple restructuring procedure for deletion

Consequently,

$$
\frac{1}{1+\frac{1}{\beta}+\frac{2 \beta-1}{\beta(s(l)+1)}} \leq B(p) \leq 1-\frac{1}{1+\frac{1}{\beta}+\frac{2 \beta-1}{\beta(s(r)+1)}}
$$

When $\beta=\sqrt{2}-1$,

$$
\frac{1}{2+\sqrt{2}+\frac{1-\sqrt{2}}{s(l)+1}} \leq B(p) \leq 1-\frac{1}{2+\sqrt{2}+\frac{1-\sqrt{2}}{s(r)+1}}
$$

If $s(p) \leq 10,0.296 \leq B(p) \leq 1-0.296$. So, every $\beta$-balanced subtree with 10 or fewer nodes is in $\mathrm{WB}(\alpha)$ for $\alpha \approx 0.296$. Similarly, every subtree with 100 or fewer nodes is in $\mathrm{WB}(\alpha)$ for $\alpha \approx 0.293$. In fact, for every fixed $k$, subtrees of size $k$ or less are in $\mathrm{WB}(\alpha)$ for $\alpha$ slightly higher than $1-\frac{1}{\sqrt{2}} \approx 0.2929$ which is the largest value of $\alpha$ for which $\mathrm{WB}(\alpha)$ trees can be maintained.

\section{BBSTs without Deletion}

In some applications of a dictionary, we need to support only the insert and search operations. In these applications, we can construct binary search trees with total cost

$$
C(T) \leq n \log _{\phi}(\sqrt{5}(n+1))
$$




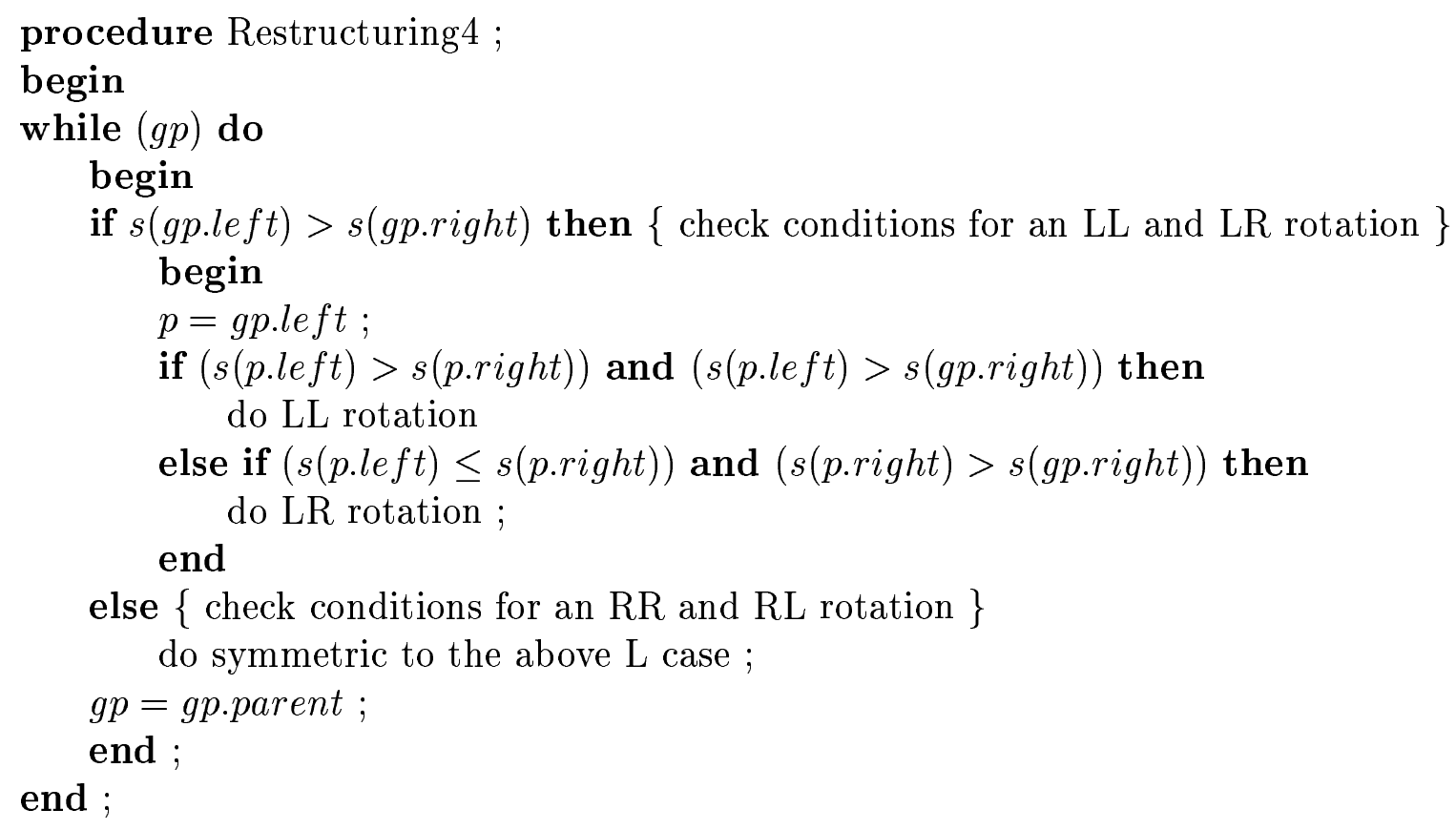

Figure 11: Simple restructuring procedure without a $\beta$ value

by using the simpler restructuring algorithm of Figure 11.

Theorem 5 When the only operations are search and insert and restructuring is done as in Figure 11, $C(T) \leq n \log _{\phi}(\sqrt{5}(n+1))$.

Proof Suppose $T$ currently has $m-1$ elements and a new element is inserted. Let $u$ be the level at which the new element is inserted. Suppose that the restructuring pass performs rotations at $q<u$ of the nodes on the path from the root to the newly inserted node. Then $C(T)$ increases by at most $v=u-q$ as a result of the insertion. The number of nodes on the path from the root to the newly inserted node at which no rotation is performed is also $v$. Let these nodes be numbered 1 through $v$ bottom to top. Let $S_{i}$ denote the number of elements in the subtree with root $i$ prior to the restructuring pass. We see that $S_{1} \geq 1$ and $S_{2} \geq 2$. For node $i, 2<i \leq v$, one of its subtrees contains node $i-1$. Without loss of generality, let this be the left subtree of $i$. Let the root of the right subtree of $i$ be $d$. So,

$$
S_{i} \geq S_{i-1}+s(d)+1
$$

If $i-1$ is not the left child of $i$, then since no rotation is done at $i, s(d) \geq S_{i-1}$. If $i-1$ is the left child of $i$, then consider node $i-2$. This is in one of the subtrees of $i$. Since no rotation is performed at $i-1, s(d) \geq S_{i-2}$. Since $S_{i-1}>S_{i-2}$, we get

$$
S_{i} \geq S_{i-1}+S_{i-2}+1
$$


Hence, $S_{v} \geq N_{v}$ where $N_{v}$ is the minimum number of elements in a COST of height $v$. So, $v \leq \log _{\phi}(\sqrt{5}(m+1))$. So, when an element is inserted into a tree that has $m-1$ elements, its cost $C(T)$ increases by at most $\log _{\phi}(\sqrt{5}(m+1))$. Starting with an empty tree and inserting $n$ elements results in a tree whose cost is at most $n \log _{\phi}(\sqrt{5}(n+1))$.

Corollary 1 The expected cost of a search or insert in a BBST constructed as above is $O(\log n)$.

Proof Since $C(T) \leq n \log _{\phi}(\sqrt{5}(n+1))$, the expected search cost is $C(T) / n \leq \log _{\phi}(\sqrt{5}(n+$ 1)). The cost of an insert is the same order as that of a search as each insert follows the corresponding search path twice (top down and bottom up).

\section{Experimental Results}

For comparison purposes, we wrote C programs for BBSTs, SBBSTs (simple BBSTs), BBSTDs (BBSTs in which procedure Restructuring4 (Figure 11) is used to restructure following inserts as well as deletes), unbalanced binary search trees (BST), AVL-trees, top-down red-black trees (RB-T), bottom-up red-black trees (RB-B) [TARJ83], weight balanced trees (WB), deterministic skip lists (DSL), treaps (TRP), and skip lists (SKIP). For the BBST and SBBST structures, we used $\beta=207 / 500$ while for the WB structure, we used $\alpha=207 / 707$. While these are not the highest permissible values of $\beta$ and $\alpha$, this choice permitted us to use integer arithmetic rather than the substantially more expensive real arithmetic. For instance, $\beta-(a, b)$ for $\beta=207 / 500$ can be checked using the comparisons $207(s(a)-1)>500 s(b)$ and $207(s(b)-1)>500 s(a)$. The randomized structures TRP and SKIP used the same random number generator with the same seed. SKIP was programmed with probability value $p=1 / 4$ as in [PUGH90].

To minimize the impact of system call overheads on run time measurements, we programmed all structures using simulated pointers (i.e., an array of nodes with integer pointers [SAHN98]). Skip lists use variable size nodes. This requires more complex storage management than required by the remaining structures which use nodes of the same size. For our experiments, we implemented skip lists using fixed size nodes, each node being of the maximum size. As a result, our run times for skip lists are smaller than if a space efficient implementation had been used. In all our tree structure implementations, null pointers were replaced by a pointer to a tail node whose data field could be set to the search/insert/delete 
key and thus avoid checking for falling off the tree. Similar tail pointers are part of the defined structure of skip and deterministic skip lists. Each tree also had a head node. WB $(\alpha)$ trees were implemented with a bottom-up restructuring pass. Our codes for SKIP and DSL are based on the codes of [PUGH90] and [PAPA93], respectively. Our AVL and RB-T codes are based on those of [PAPA93] and [SEDG94]. The treap structure was implemented using joins and splits rather than rotations. This results in better performance. Furthermore, AVL, RB-B, WB, and BBST were implemented with parent pointers in addition to left and right child pointers. For BBSTs, the enhancements described in Section 4.4 for insert and delete (see Figure 8) were employed. No rotations were performed during a search when using any of the structures. Our codes are available from www.cise.ufl.edu/ sahni/search.zip.

We experimented with $n=10,000,50,000,100,000$, and 200,000. For each $n$, the following experiments were conducted:

(a) start with an empty structure and perform $n$ inserts;

(b) search for each item in the resulting structure once; items are searched for in the order they were inserted

(c) perform an alternating sequence of $n$ inserts and $n$ deletes; in this, the $n$ elements inserted in (a) are deleted in the order they were inserted and $n$ new elements are inserted

(d) search for each of the remaining $n$ elements in the order they were inserted

(e) delete the $n$ elements in the order they were inserted.

For each $n$, the above five part experiment was repeated ten times using different random permutations of distinct elements. For each permutation, we measured the total number of element comparisons performed and then averaged these over the ten permutations.

First, we report on the relative performance of SBBSTs, BBSTDs, and BBSTs. Table 1 gives the average number of key comparisons performed for each of the five parts of the experiment. The three versions of our proposed data structure are very competitive on this measure. BBSTDs and BBSTs generally performed fewer comparisons than did SBBSTs. All three structures had a comparison count within $2 \%$ of one another. However, when we used ordered data rather than random data (Table 2), SBBSTs performed noticeably inferior to BBSTDs and BBSTs; the latter two remained very competitive.

Tables 3 and 4 give the average heights of the trees using random data and using ordered data, respectively. The first number gives the height following part (a) of the experiment and the second following part (c). The numbers are identical for BBSTDs and BBSTs and slightly higher (lower) for SBBSTs using random (ordered) data.

The average number of rotations performed by each of the three structures is given in Tables 5 and 6. A single rotation (i.e., LL or RR) is denoted ' $\mathrm{S}$ ' and a double rotation (i.e., LR or RL) denoted ' $\mathrm{D}$ '. In the case of BBSTs, double rotations have been divided into 


\begin{tabular}{|c|c||c|c|c|}
\hline \hline$n$ & operation & SBBST & BBSTD & BBST \\
\hline \hline \multirow{5}{*}{10,000} & insert & 212495 & 212223 & 212111 \\
& search & 194661 & 191599 & 191578 \\
& ins/del & 416125 & 416967 & 416862 \\
& search & 194957 & 191666 & 191676 \\
& delete & 168033 & 166441 & 166487 \\
\hline \multirow{5}{*}{50,000} & insert & 1241080 & 1236682 & 1236114 \\
& search & 1152137 & 1135131 & 1134969 \\
& ins/del & 2437918 & 2438083 & 2437639 \\
& search & 1153821 & 1134277 & 1134062 \\
& delete & 1018675 & 1007766 & 1007688 \\
\hline \multirow{5}{*}{100,000} & insert & 2635913 & 2624829 & 2623792 \\
& search & 2458079 & 2423988 & 2423613 \\
& ins/del & 5183619 & 5180383 & 5179653 \\
& search & 2461221 & 2420282 & 2419990 \\
& delete & 2190798 & 2168049 & 2168110 \\
\hline \multirow{5}{*}{200,000} & insert & 5580139 & 5555190 & 5553256 \\
& search & 5223989 & 5148220 & 5147698 \\
& ins/del & 10981441 & 10969578 & 10968053 \\
& search & 5229172 & 5144808 & 5144148 \\
& delete & 4692447 & 4641349 & 4641389 \\
\hline \hline
\end{tabular}

Table 1: The number of key comparisons on random inputs 


\begin{tabular}{|c|c||c|c|c|}
\hline \hline$n$ & operation & SBBST & BBSTD & BBST \\
\hline \hline \multirow{5}{*}{10,000} & insert & 170182 & 150554 & 150554 \\
& search & 188722 & 185530 & 185530 \\
& ins/del & 425305 & 315177 & 314998 \\
& search & 191681 & 184155 & 184155 \\
& delete & 215214 & 135311 & 135131 \\
\hline \multirow{5}{*}{50,000} & insert & 991526 & 872967 & 872967 \\
& search & 1117174 & 1101481 & 1101481 \\
& ins/del & 2472808 & 1806346 & 1805439 \\
& search & 1116390 & 1098065 & 1098065 \\
& delete & 1277756 & 792717 & 791815 \\
\hline \multirow{5}{*}{100,000} & insert & 2103808 & 1850548 & 1850548 \\
& search & 2384327 & 2354757 & 2354757 \\
& ins/del & 5249194 & 3823415 & 3821594 \\
& search & 2382759 & 2346118 & 2346128 \\
& delete & 2738294 & 1686397 & 1684584 \\
\hline & insert & 4449143 & 3903083 & 3903083 \\
& search & 5068632 & 4946753 & 4946753 \\
200,000 & ins/del & 11105525 & 8051695 & 8048058 \\
& search & 5065496 & 5001967 & 5001967 \\
& delete & 5842168 & 3580856 & 3577223 \\
\hline \hline
\end{tabular}

Table 2: The number of key comparisons on ordered inputs

\begin{tabular}{|c||c|c|c|}
\hline \hline$n$ & SBBST & BBSTD & BBST \\
\hline \hline 10,000 & 17,17 & 16,16 & 16,16 \\
50,000 & 20,20 & 19,19 & 19,19 \\
100,000 & 21,21 & 20,20 & 20,20 \\
200,000 & 22,23 & 21,21 & 21,21 \\
\hline \hline
\end{tabular}

Table 3: Height of the trees on random inputs

\begin{tabular}{|c||c|c|c|}
\hline \hline$n$ & SBBST & BBSTD & BBST \\
\hline \hline 10,000 & 16,15 & 17,17 & 17,17 \\
50,000 & 20,20 & 20,20 & 20,20 \\
100,000 & 21,21 & 21,21 & 21,21 \\
200,000 & 22,22 & 23,22 & 23,22 \\
\hline \hline
\end{tabular}

Table 4: Height of the trees on ordered inputs 


\begin{tabular}{|c|c||c|c|c|c|c|c|c|c|}
\hline \hline \multirow{2}{*}{$n$} & \multicolumn{1}{|c||}{} & \multicolumn{2}{c|}{ SBBST } & \multicolumn{2}{c|}{ BBSTD } & \multicolumn{4}{c|}{ BBST } \\
\cline { 3 - 9 } & operation & $\mathrm{S}$ & $\mathrm{D}$ & $\mathrm{S}$ & $\mathrm{D}$ & $\mathrm{S}$ & $\mathrm{D}$ & $\mathrm{DS}$ & $\mathrm{DD}$ \\
\hline \hline \multirow{3}{*}{10,000} & insert & 2341 & 2220 & 5045 & 4314 & 5025 & 3938 & 151 & 93 \\
& ins/del & 4269 & 3216 & 10158 & 6311 & 10104 & 5849 & 232 & 103 \\
& delete & 1607 & 1110 & 5235 & 2104 & 5201 & 2018 & 51 & 28 \\
\hline \multirow{3}{*}{50,000} & insert & 11719 & 11120 & 25216 & 21596 & 25059 & 19732 & 754 & 455 \\
& ins/del & 21330 & 16125 & 51238 & 31499 & 50979 & 29198 & 1161 & 531 \\
& delete & 8058 & 5648 & 26214 & 10462 & 26068 & 10033 & 248 & 131 \\
\hline \multirow{3}{*}{100,000} & insert & 23450 & 22262 & 50283 & 43230 & 50047 & 39461 & 1527 & 920 \\
& ins/del & 42780 & 32203 & 102218 & 62967 & 101836 & 58491 & 2275 & 1046 \\
& delete & 16095 & 11306 & 52227 & 21022 & 51943 & 20147 & 496 & 260 \\
\hline \multirow{3}{*}{200,000} & insert & 46934 & 44525 & 100664 & 86605 & 100205 & 79013 & 3054 & 1840 \\
& ins/del & 85283 & 64417 & 204459 & 125960 & 203568 & 116940 & 4593 & 2059 \\
& delete & 32233 & 22551 & 104344 & 41884 & 103826 & 40157 & 990 & 523 \\
\hline \hline
\end{tabular}

Table 5: The number of rotations on random inputs

three categories: $\mathrm{D}=\mathrm{LR}$ and $\mathrm{RL}$ rotations that do not perform a second substep rotation; $\mathrm{DS}=\mathrm{LR}$ and RL rotations with a second substep rotation of type LL and RR; DD $=\mathrm{LR}$ and RL rotations with a second substep rotation of type LR and RL. BBSTDs and BBSTs performed a comparable number of rotations on both data sets. However, on random data SBBSTs performed about half as many rotations as did BBSTDs and BBSTs. On ordered data, SBBSTs performed 15 to $20 \%$ fewer rotations on part (a), $34 \%$ fewer on part (c), and $51 \%$ fewer on part (e).

The run-time performance of the structures is significantly influenced by compiler and architectural features as well as the complexity of a key comparison. The results we report are from a SUN SPARC-5 using the UNIX C compiler cc with optimization option. Because of instruction pipelining features, cache replacement policies, etc., the measured run times are not always consistent with the compiler and architecture independent metrics reported in Tables 1 through 6 and later in Tables 9 through 14. For example, since the search codes for all tree based methods are essentially identical, we would expect methods with a smaller comparison count to have a smaller run time for parts (b) and (d) of the experiment. This was not always the case.

Tables 7 and 8 give the run times of the three BBST structures using real (i.e., floating point) keys. The sum of the run times for parts (a) through (e) of the experiment is graphed in Figure 12. For random data, SBBSTs significantly and consistently outperformed BBSTDs and BBSTs. On ordered data, however, BBSTDs were slightly faster than BBSTs and both 


\begin{tabular}{|c|c||c|c|c|c|c|c|c|c|}
\hline \hline \multirow{2}{*}{$n$} & \multicolumn{1}{|c||}{} & \multicolumn{2}{c|}{ SBBST } & \multicolumn{2}{c|}{ BBSTD } & \multicolumn{4}{c|}{ BBST } \\
\cline { 3 - 9 } & operation & $\mathrm{S}$ & $\mathrm{D}$ & $\mathrm{S}$ & $\mathrm{D}$ & $\mathrm{S}$ & $\mathrm{D}$ & $\mathrm{DS}$ & $\mathrm{DD}$ \\
\hline \hline \multirow{3}{*}{10,000} & insert & 9984 & 0 & 9985 & 2387 & 9985 & 2387 & 0 & 0 \\
& ins/del & 14997 & 0 & 16567 & 6130 & 16644 & 5797 & 25 & 154 \\
& delete & 4989 & 0 & 6570 & 3726 & 6647 & 3392 & 26 & 154 \\
\hline \multirow{3}{*}{50,000} & insert & 49980 & 0 & 49983 & 11956 & 49983 & 11956 & 0 & 0 \\
& ins/del & 74996 & 0 & 82862 & 30659 & 83247 & 28982 & 137 & 770 \\
& delete & 24987 & 0 & 32859 & 18686 & 33242 & 17018 & 136 & 766 \\
\hline \multirow{3}{*}{100,000} & insert & 99979 & 0 & 99983 & 23917 & 99983 & 23917 & 0 & 0 \\
& ins/del & 149996 & 0 & 165738 & 61327 & 166504 & 57969 & 280 & 1540 \\
& delete & 49986 & 0 & 65733 & 37392 & 66505 & 34040 & 278 & 1536 \\
\hline \multirow{3}{*}{200,000} & insert & 199978 & 0 & 199982 & 47839 & 199982 & 47839 & 0 & 0 \\
& ins/del & 299996 & 0 & 331473 & 122653 & 333012 & 115938 & 559 & 3078 \\
& delete & 99985 & 0 & 131478 & 74795 & 133016 & 68086 & 557 & 3076 \\
\hline \hline
\end{tabular}

Table 6: The number of rotations on ordered inputs

were significantly faster than SBBSTs.

Since BBSTs generated trees with the least search cost, we expect BBSTs to outperform SBBSTs and BBSTDs in applications where the comparison cost is very high relative to that of other operations and searches are done with a much higher frequency than inserts and deletes. However, with the mix of operations used in our tests, SBBSTs are the clear choice for random inputs and BBSTDs for ordered inputs.

In comparing with the other structures, our tables repeat the data for BBSTs. The reader may make the comparison with SBBSTs and BBSTDs.

The average number of comparisons for each of the five parts of the experiment are given in Table 9. On the comparison measure, AVL, RB-B, WB, and BBSTs are the front runners and are quite competitive with one another. On parts (a) (insert $n$ elements) and (c) (insert $n$ and delete $n$ elements), AVL trees performed best while on the two search tests ((b) and (d)) and the deletion test (e), BBSTs performed best.

Table 10 gives the number of comparisons performed when ordered data (i.e., the elements in part (a) are $1,2, \ldots, n$ and are inserted in this order) and those in part (c) are $n+1, \ldots, 2 n$ (in this order) is used instead of random permutations of distinct elements. This experiment attempts to model realistic situations in which the inserted elements are in "nearly sorted order". BSTs were not included in this test as they perform very poorly with ordered data taking $\mathrm{O}\left(n^{2}\right)$ time to insert $n$ times. The computer time needed to perform this test on BSTs was determined to be excessive. This test exhibited greater variance in performance. 


\begin{tabular}{|c|c||c|c|c|}
\hline \hline$n$ & operation & SBBST & BBSTD & BBST \\
\hline \hline \multirow{5}{*}{10,000} & insert & 0.23 & 0.34 & 0.36 \\
& search & 0.07 & 0.10 & 0.10 \\
& ins/del & 0.44 & 0.75 & 0.79 \\
& search & 0.08 & 0.10 & 0.10 \\
& delete & 0.17 & 0.29 & 0.30 \\
\hline \multirow{5}{*}{50,000} & insert & 1.43 & 1.76 & 1.93 \\
& search & 0.47 & 0.53 & 0.52 \\
& ins/del & 2.76 & 3.89 & 4.22 \\
& search & 0.50 & 0.54 & 0.55 \\
& delete & 1.13 & 1.62 & 1.76 \\
\hline \multirow{5}{*}{100,000} & insert & 2.96 & 3.94 & 4.36 \\
& search & 1.08 & 1.17 & 1.16 \\
& ins/del & 6.11 & 8.58 & 9.30 \\
& search & 1.12 & 1.20 & 1.22 \\
& delete & 2.50 & 3.66 & 3.95 \\
\hline \multirow{3}{*}{200,000} & insert & 6.85 & 8.92 & 9.33 \\
& search & 2.41 & 2.58 & 2.57 \\
& ins/del & 13.86 & 19.49 & 20.46 \\
& search & 2.49 & 2.69 & 2.66 \\
& delete & 5.61 & 8.25 & 8.80 \\
\hline \hline
\end{tabular}

Time Unit : sec

Table 7: Run time on random real inputs 


\begin{tabular}{|c|c||c|c|c|}
\hline \hline$n$ & operation & SBBST & BBSTD & BBST \\
\hline \hline \multirow{5}{*}{10,000} & insert & 0.27 & 0.23 & 0.20 \\
& search & 0.08 & 0.07 & 0.07 \\
& ins/del & 0.53 & 0.50 & 0.43 \\
& search & 0.08 & 0.07 & 0.05 \\
& delete & 0.18 & 0.23 & 0.20 \\
\hline \multirow{5}{*}{50,000} & insert & 1.43 & 1.25 & 1.12 \\
& search & 0.40 & 0.30 & 0.30 \\
& ins/del & 2.80 & 2.17 & 2.37 \\
& search & 0.40 & 0.30 & 0.30 \\
& delete & 1.07 & 0.90 & 0.97 \\
\hline \multirow{5}{*}{100,000} & insert & 3.28 & 2.58 & 2.77 \\
& search & 0.90 & 0.62 & 0.63 \\
& ins/del & 6.15 & 4.70 & 5.13 \\
& search & 0.87 & 0.62 & 0.63 \\
& delete & 2.35 & 1.93 & 2.10 \\
\hline \multirow{3}{*}{200,000} & insert & 7.37 & 4.55 & 4.92 \\
& search & 1.85 & 1.32 & 1.32 \\
& ins/del & 13.35 & 10.03 & 10.93 \\
& search & 1.87 & 1.33 & 1.33 \\
& delete & 5.08 & 4.17 & 4.43 \\
\hline \hline
\end{tabular}

Time Unit : sec

Table 8: Run time on ordered real inputs 


\begin{tabular}{|c|c||c|c|c|c|c|c|c|c|c|}
\hline \hline$n$ & operation & BST & AVL & RB-T & RB-B & WB & BBST & DSL & TRP & SKIP \\
\hline \hline \multirow{5}{*}{10,000} & insert & 264175 & 211401 & 262838 & 211886 & 211916 & 212111 & 276247 & 296866 & 224757 \\
& search & 254175 & 193253 & 194606 & 194291 & 194153 & 191578 & 258089 & 258662 & 255072 \\
& ins/del & 516853 & 411220 & 515184 & 414990 & 414635 & 416862 & 923524 & 601137 & 519430 \\
& search & 252200 & 193141 & 197399 & 195525 & 194442 & 191676 & 256578 & 254119 & 256124 \\
& delete & 215555 & 167312 & 200218 & 167455 & 167531 & 166487 & 526242 & 242743 & 231745 \\
\hline \multirow{5}{*}{50,000} & insert & 1560958 & 1234911 & 1550701 & 1236968 & 1238628 & 1236114 & 1640660 & 1717037 & 1357076 \\
& search & 1510958 & 1147273 & 1150466 & 1146754 & 1149970 & 1134969 & 1512093 & 1503452 & 1537547 \\
& ins/del & 3061868 & 2417733 & 3058045 & 2424944 & 2431281 & 2437639 & 5351715 & 3456045 & 2996512 \\
& search & 1500504 & 1145808 & 1173662 & 1152764 & 1151578 & 1134062 & 1499657 & 1497081 & 1501731 \\
& delete & 1316917 & 1013535 & 1242426 & 1013144 & 1015988 & 1007688 & 3077266 & 1451835 & 1373858 \\
\hline \multirow{5}{*}{100,000} & insert & 3329780 & 2623894 & 3305332 & 2626314 & 2631411 & 2623792 & 3513401 & 3632046 & 2919371 \\
& search & 3229780 & 2445659 & 2451137 & 2446466 & 2453855 & 2423613 & 3244497 & 3247143 & 3188621 \\
& ins/del & 6537563 & 5137280 & 6564352 & 5154118 & 5170695 & 5179653 & 11545200 & 7476441 & 6399463 \\
& search & 3208453 & 2443038 & 2502098 & 2457531 & 2456748 & 2419990 & 3229747 & 3310823 & 3225343 \\
& delete & 2839934 & 2181327 & 2692672 & 2177946 & 2185213 & 2168110 & 6561272 & 3177135 & 2981173 \\
\hline & insert & 7076132 & 5553640 & 7016676 & 5558174 & 5571133 & 5553256 & 7483199 & 7682439 & 6178596 \\
& search & 6876132 & 5191730 & 5209189 & 5199786 & 5215568 & 5147698 & 6887196 & 6797942 & 6697223 \\
& ins/del & 13907058 & 10862426 & 13940982 & 10921880 & 10956496 & 10968053 & 24207106 & 15543559 & 13377747 \\
& search & 6830718 & 5186737 & 5332771 & 5223154 & 5220965 & 5144148 & 6814733 & 6916150 & 6680642 \\
& delete & 6095324 & 4664876 & 5800203 & 4664344 & 4680768 & 4641389 & 13811271 & 6700557 & 6149268 \\
\hline \hline
\end{tabular}

Table 9: The number of key comparisons on random inputs 


\begin{tabular}{|c|c||c|c|c|c|c|c|c|c|}
\hline \hline$n$ & operation & AVL & RB-T & RB-B & WB & BBST & DSL & TRP & SKIP \\
\hline \hline \multirow{5}{*}{10,000} & insert & 277234 & 376228 & 241383 & 171017 & 150554 & 435199 & 135989 & 247129 \\
& search & 191917 & 188246 & 190106 & 188722 & 185530 & 262423 & 271087 & 256706 \\
& ins/del & 421032 & 718040 & 508810 & 425843 & 314998 & 983676 & 390899 & 354566 \\
& search & 195133 & 189494 & 190090 & 191681 & 184155 & 249694 & 269031 & 250538 \\
& delete & 104038 & 276136 & 218216 & 214930 & 135131 & 468244 & 193080 & 84392 \\
\hline \multirow{5}{*}{50,000} & insert & 1618930 & 2233658 & 1436225 & 995720 & 872967 & 2585557 & 825390 & 1422120 \\
& search & 1120497 & 1117001 & 1120495 & 1117174 & 1101481 & 1509152 & 1540082 & 1467217 \\
& ins/del & 2418422 & 4311748 & 3055100 & 2475487 & 1805439 & 6019215 & 2194668 & 1973416 \\
& search & 1124001 & 1168633 & 1126126 & 1116390 & 1098065 & 1481819 & 1568903 & 1449810 \\
& delete & 607478 & 1719212 & 1323918 & 1276262 & 791815 & 2785792 & 1181612 & 486498 \\
\hline \multirow{5}{*}{100,000} & insert & 3437858 & 4767564 & 3072389 & 2112201 & 1850548 & 5521408 & 1724473 & 2925618 \\
& search & 2390963 & 2383979 & 2390961 & 2384327 & 2354757 & 3218246 & 3564282 & 2970715 \\
& ins/del & 5111850 & 9223606 & 6510188 & 5254541 & 3821594 & 12788447 & 4438266 & 4406427 \\
& search & 2397971 & 2487243 & 2402224 & 2382759 & 2346128 & 3163554 & 3281308 & 3277089 \\
& delete & 1289954 & 3737982 & 2847792 & 2735270 & 1684584 & 5971196 & 2403622 & 961283 \\
\hline \multirow{5}{*}{200,000} & insert & 7275714 & 10135418 & 6544713 & 4465935 & 3903083 & 11743159 & 3428355 & 6403207 \\
& search & 5081893 & 5067933 & 5081891 & 5068632 & 4946753 & 6836428 & 7174727 & 6448304 \\
& ins/del & 10773706 & 19647336 & 13820364 & 11116226 & 8048058 & 27076911 & 9054078 & 9062233 \\
& search & 5095909 & 5274461 & 5104418 & 5065496 & 5001967 & 6727017 & 7006341 & 6458321 \\
& delete & 2729906 & 8075474 & 6095538 & 5836096 & 3577223 & 12741948 & 5094044 & 1995215 \\
\hline \hline
\end{tabular}

Table 10: The number of key comparisons on ordered inputs 


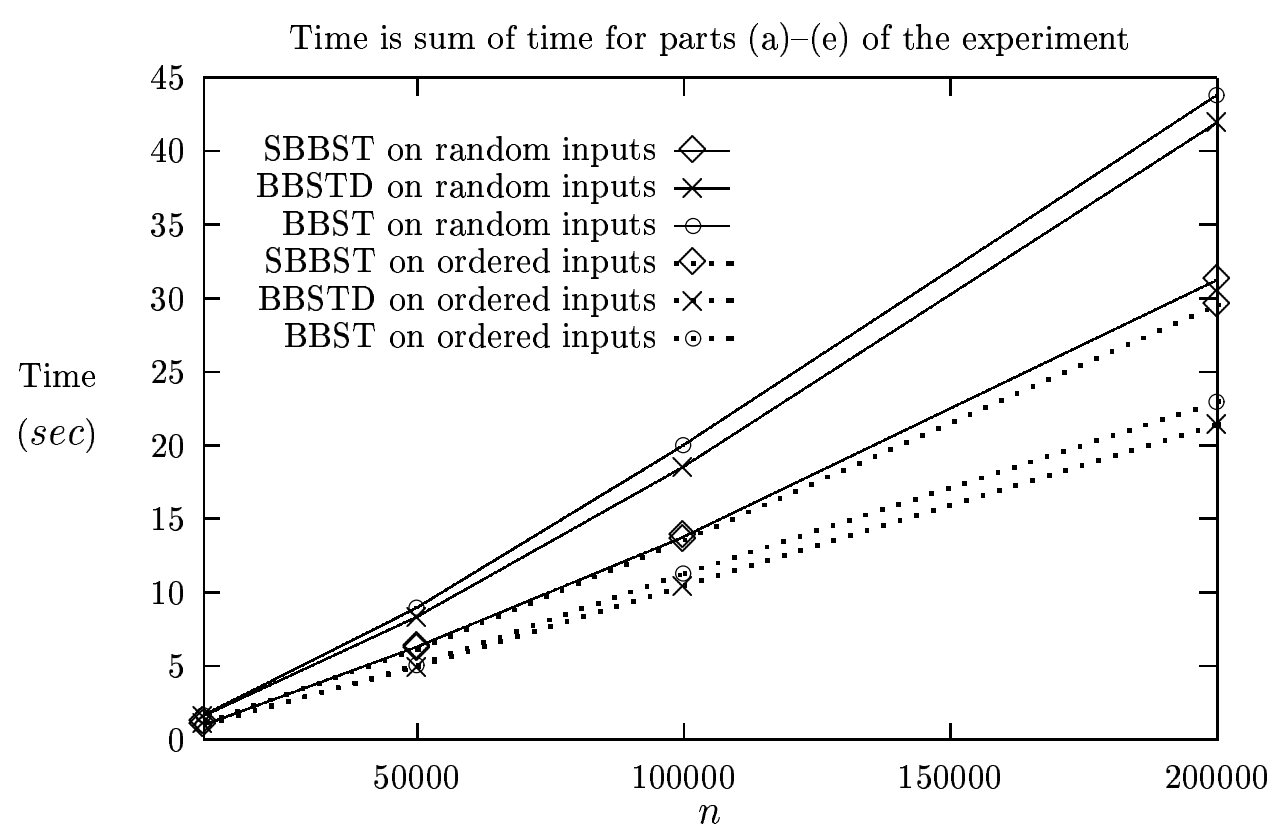

Figure 12: Run time on real inputs

Among the deterministic structures, BBSTs outperformed the others in parts (a) - (d) while AVL trees were ahead in part (e). For part (a), BBSTs performed approximately $45 \%$ fewer comparisons than did AVL trees and approximately $12 \%$ fewer than WB trees. The randomized structure TRP was the best of the eight structures reported in Table 10 for part (a). It performed approximately $10 \%$ fewer comparisons than did BBST trees. However, the BBST remained best overall on parts (b), (c), and (d).

The heights of the trees (number of levels in the case of DSL and SKIP) for the experiments with random and ordered data are given in Tables 11 and 12 respectively. The first number in each table entry is the tree height after part (a) of the experiment and the second, the height after part (c). In all cases, the number of levels using skip lists is fewest. However, among the tree structures, AVL and BBST trees have least height on random data and AVL has least with ordered data. This latter result is to be expected because when elements are inserted in sorted order into an AVL tree, the tree height is $\log _{2}(n+1)$; that is, the tree height is the minimum possible.

Tables 13 and 14, respectively, give the number of rotations performed by each of the deterministic tree schemes for experiment parts (a), (c), and (e). Note that none of the schemes performs rotations during a search.

On ordered data, BBSTs perform about $25 \%$ more rotations than do the remaining structures. These remaining structures perform about the same number of rotations. On 


\begin{tabular}{|c||c|c|c|c|c|c|c|c|c|}
\hline \hline$n$ & BST & AVL & RB-T & RB-B & WB & BBST & DSL & TRP & SKIP \\
\hline \hline 10,000 & 31,31 & 16,16 & 17,18 & 16,17 & 17,17 & 16,16 & 12,11 & 32,31 & 8,8 \\
50,000 & 38,38 & 19,19 & 20,21 & 19,20 & 20,20 & 19,19 & 13,12 & 38,37 & 9,9 \\
100,000 & 41,41 & 20,20 & 21,22 & 20,21 & 21,22 & 20,20 & 14,13 & 41,40 & 9,9 \\
200,000 & 44,43 & 21,21 & 22,24 & 21,22 & 23,23 & 21,21 & 15,14 & 43,44 & 9,9 \\
\hline \hline
\end{tabular}

Table 11: Height of the trees on random inputs

\begin{tabular}{|c||c|c|c|c|c|c|c|c|}
\hline \hline$n$ & AVL & RB-T & RB-B & WB & BBST & DSL & TRP & SKIP \\
\hline \hline 10,000 & 14,14 & 20,20 & 24,24 & 16,15 & 17,17 & 14,13 & 33,34 & 8,8 \\
50,000 & 16,16 & 23,23 & 29,28 & 20,20 & 20,20 & 16,16 & 41,41 & 9,9 \\
100,000 & 17,17 & 25,25 & 31,30 & 21,21 & 21,21 & 17,17 & 46,41 & 9,9 \\
200,000 & 18,18 & 27,27 & 33,32 & 22,22 & 23,22 & 18,18 & 47,46 & 9,9 \\
\hline \hline
\end{tabular}

Table 12: Height of the trees on ordered inputs

random data, AVL trees, bottom-up red-black trees and WB trees perform a comparable number of rotations. Top-down red-black trees and BBST trees perform a significantly larger number of rotations. In fact, BBSTs perform about twice as many rotations as AVL trees. The AVL-tree data reported in Table 13 is consistent with the finding in [KARL76] that random inserts require no rotations approximately $53 \%$ of the time, a single rotation approximately $23 \%$ of the time, and a double rotation approximately $23 \%$ of the time (these percentages do not add to $100 \%$ because of rounding errors).

The average run times for the random data tests are given in Table 15 and in Table 16 for the ordered data test. The sum of the run time for parts (b) and (d) of the experiment are graphed in Figure 13 for random data and on Figure 14 for ordered data. The graph of Figure 13 shows only one line MIX for AVL, RB-T, RB-B, and WB as the times for these are fairly close. The five balanced binary tree structures are quite competitive with respect to search operations (i.e., parts (b) and (d)). RB-B generally outperformed the other structures on parts (a), (c), and (e). Using ordered real keys, the treap was the clear winner on parts (a), (c), and (e) while BBSTs handily outperformed the remaining structures on parts (b) and (d). 


\begin{tabular}{|c|c|c|c|c|c|c|c|c|c|c|c|c|c|}
\hline \multirow[b]{2}{*}{$n$} & \multirow[b]{2}{*}{ operation } & \multicolumn{2}{|c|}{$\overline{\overline{A V L}}$} & \multicolumn{2}{|c|}{$\overline{\overline{\text { RB-T }}}$} & \multicolumn{2}{|c|}{$\overline{\mathrm{RB}-\mathrm{B}}$} & \multicolumn{2}{|c|}{$\overline{\mathrm{WB}}$} & \multicolumn{4}{|c|}{$\overline{\overline{\mathrm{BBST}}}$} \\
\hline & & $\mathrm{S}$ & $\mathrm{D}$ & $\mathrm{S}$ & $\mathrm{D}$ & $\mathrm{S}$ & $\mathrm{D}$ & $\mathrm{S}$ & $\mathrm{D}$ & $\mathrm{S}$ & $\mathrm{D}$ & $\mathrm{DS}$ & $\mathrm{DD}$ \\
\hline \multirow{3}{*}{10,000} & insert & 2328 & 2322 & $\bar{~} 1964$ & 1955 & 1946 & 1933 & 22274 & 2065 & $\bar{~} 5025$ & "3938 & $\overline{151}$ & 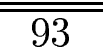 \\
\hline & del & 4343 & 3224 & 14773 & 8213 & 4053 & 2591 & 4256 & 2978 & 10104 & 5849 & 232 & 103 \\
\hline & delete & 1645 & 1120 & 9558 & 2678 & 1845 & 1166 & 1595 & 1022 & 5201 & 2018 & 51 & 28 \\
\hline \multirow{3}{*}{50,000} & insert & 11664 & 11614 & 9822 & 9815 & 9710 & 9689 & 11355 & 10352 & 25059 & 19732 & 754 & 455 \\
\hline & ins/del & 21585 & 16214 & 81895 & 45180 & 20255 & 12979 & 21266 & 14975 & 50979 & 29198 & 1161 & 531 \\
\hline & delete & 8231 & 5630 & 54806 & 13431 & 9196 & 5844 & 7963 & 5194 & 26068 & 10033 & 248 & 131 \\
\hline \multirow{3}{*}{100,000} & insert & 23316 & 23254 & 19593 & 19677 & 19340 & 19414 & 22723 & 20730 & 50047 & 39461 & 1527 & 920 \\
\hline & ins/del & 43243 & 32361 & 196769 & 103835 & 40618 & 25919 & 42567 & 29898 & 101836 & 58491 & 2275 & 1046 \\
\hline & delete & 16466 & 11264 & 119825 & 26953 & 18530 & 11708 & 16024 & 10420 & 51943 & 20147 & 496 & 260 \\
\hline \multirow{3}{*}{200,000} & insert & 46631 & 46518 & 39290 & 39291 & 38797 & 38793 & 45458 & 41480 & 100205 & 79013 & 3054 & 1840 \\
\hline & ins/del & 86218 & 64712 & 394187 & 209941 & 80892 & 52030 & 84927 & 59911 & 203568 & 116940 & 4593 & 2059 \\
\hline & delete & 33047 & 22477 & 247905 & 54046 & 37083 & 23379 & 31984 & 20800 & 103826 & 40157 & 990 & 523 \\
\hline
\end{tabular}

Table 13: The number of rotations on random inputs 


\begin{tabular}{|c|c||c|c|c|c|c|c|c|c|c|c|c|c|}
\hline \hline \multirow{3}{*}{$n$} & \multicolumn{3}{|c||}{} & \multicolumn{2}{c|}{ AVL } & \multicolumn{2}{c|}{ RB-T } & \multicolumn{2}{c|}{ RB-B } & \multicolumn{2}{c|}{ WB } & \multicolumn{4}{c|}{ BBST } \\
\cline { 3 - 12 } & operation & $\mathrm{S}$ & $\mathrm{D}$ & $\mathrm{S}$ & $\mathrm{D}$ & $\mathrm{S}$ & $\mathrm{D}$ & $\mathrm{S}$ & $\mathrm{D}$ & $\mathrm{S}$ & $\mathrm{D}$ & $\mathrm{DS}$ & $\mathrm{DD}$ \\
\hline \hline \multirow{3}{*}{10,000} & insert & 9986 & 0 & 9980 & 0 & 9976 & 0 & 9984 & 0 & 9985 & 2387 & 0 & 0 \\
& ins/del & 14996 & 0 & 14999 & 0 & 14995 & 0 & 14997 & 0 & 16644 & 5797 & 25 & 154 \\
& delete & 4990 & 0 & 4983 & 1 & 4989 & 0 & 4989 & 0 & 6647 & 3392 & 26 & 154 \\
\hline \multirow{3}{*}{50,000} & insert & 49984 & 0 & 49977 & 0 & 49971 & 0 & 49980 & 0 & 49983 & 11956 & 0 & 0 \\
& ins/del & 74994 & 0 & 75000 & 0 & 74994 & 0 & 74996 & 0 & 83247 & 28982 & 137 & 770 \\
& delete & 24988 & 0 & 24978 & 1 & 24986 & 0 & 24987 & 0 & 33242 & 17018 & 136 & 766 \\
\hline \multirow{3}{*}{100,000} & insert & 99983 & 0 & 99975 & 0 & 99969 & 0 & 99979 & 0 & 99983 & 23917 & 0 & 0 \\
& ins/del & 149994 & 0 & 150000 & 0 & 149994 & 0 & 149996 & 0 & 166504 & 57969 & 280 & 1540 \\
& delete & 49987 & 0 & 49977 & 1 & 49985 & 0 & 49986 & 0 & 66505 & 34040 & 278 & 1536 \\
\hline \multirow{3}{*}{200,000} & insert & 199982 & 0 & 199973 & 0 & 199967 & 0 & 199978 & 0 & 199982 & 47839 & 0 & 0 \\
& ins/del & 299994 & 0 & 300000 & 0 & 299994 & 0 & 299996 & 0 & 333012 & 115938 & 559 & 3078 \\
& delete & 99986 & 0 & 99976 & 1 & 99984 & 0 & 99985 & 0 & 133016 & 68086 & 557 & 3076 \\
\hline \hline
\end{tabular}

Table 14: The number of rotations on ordered inputs 


\begin{tabular}{|c|c||c|c|c|c|c|c|c|c|c|}
\hline \hline$n$ & operation & BST & AVL & RB-T & RB-B & WB & BBST & DSL & TRP & SKIP \\
\hline \hline \multirow{5}{*}{10,000} & insert & 0.14 & 0.15 & 0.21 & 0.17 & 0.23 & 0.36 & 0.22 & 0.23 & 0.30 \\
& search & 0.09 & 0.07 & 0.09 & 0.10 & 0.08 & 0.10 & 0.13 & 0.13 & 0.21 \\
& ins/del & 0.24 & 0.27 & 0.51 & 0.32 & 0.38 & 0.79 & 0.62 & 0.41 & 0.53 \\
& search & 0.09 & 0.08 & 0.09 & 0.10 & 0.08 & 0.10 & 0.12 & 0.12 & 0.21 \\
& delete & 0.09 & 0.09 & 0.17 & 0.14 & 0.14 & 0.30 & 0.28 & 0.11 & 0.19 \\
\hline \multirow{5}{*}{50,000} & insert & 0.94 & 0.97 & 1.22 & 0.86 & 1.29 & 1.93 & 1.48 & 1.19 & 1.67 \\
& search & 0.64 & 0.52 & 0.50 & 0.51 & 0.51 & 0.52 & 0.87 & 0.71 & 1.44 \\
& ins/del & 1.68 & 1.77 & 2.74 & 1.53 & 2.29 & 4.22 & 3.93 & 2.17 & 3.15 \\
& search & 0.66 & 0.55 & 0.56 & 0.54 & 0.56 & 0.55 & 0.86 & 0.71 & 1.33 \\
& delete & 0.63 & 0.67 & 1.10 & 0.72 & 0.92 & 1.76 & 1.80 & 0.69 & 1.22 \\
\hline \multirow{5}{*}{100,000} & insert & 2.06 & 1.85 & 2.34 & 1.90 & 2.66 & 4.36 & 3.05 & 2.67 & 3.61 \\
& search & 1.43 & 1.13 & 1.09 & 1.13 & 1.14 & 1.16 & 1.84 & 1.66 & 3.00 \\
& ins/del & 3.63 & 3.93 & 6.18 & 3.33 & 4.96 & 9.30 & 8.45 & 4.84 & 7.10 \\
& search & 1.45 & 1.26 & 1.27 & 1.17 & 1.26 & 1.22 & 1.83 & 1.65 & 3.01 \\
& delete & 1.39 & 1.50 & 2.51 & 1.55 & 2.03 & 3.95 & 3.91 & 1.61 & 2.75 \\
\hline \multirow{5}{*}{200,000} & insert & 4.34 & 3.95 & 5.20 & 3.88 & 5.56 & 9.33 & 6.77 & 5.81 & 7.90 \\
& search & 3.19 & 2.49 & 2.42 & 2.50 & 2.45 & 2.57 & 4.14 & 3.67 & 6.62 \\
& ins/del & 8.01 & 8.25 & 13.78 & 7.29 & 10.65 & 20.46 & 18.88 & 10.48 & 15.83 \\
& search & 3.21 & 2.83 & 2.86 & 2.62 & 2.74 & 2.66 & 4.08 & 3.73 & 6.74 \\
& delete & 3.11 & 3.27 & 5.55 & 3.41 & 4.43 & 8.80 & 8.56 & 3.54 & 6.04 \\
\hline \hline
\end{tabular}

Time Unit : sec

Table 15: Run time on random real inputs 


\begin{tabular}{|c|c||c|c|c|c|c|c|c|c|}
\hline \hline$n$ & operation & AVL & RB-T & RB-B & WB & BBST & DSL & TRP & SKIP \\
\hline \hline \multirow{5}{*}{10,000} & insert & 0.13 & 0.22 & 0.15 & 0.25 & 0.20 & 0.25 & 0.12 & 0.30 \\
& search & 0.07 & 0.08 & 0.07 & 0.07 & 0.07 & 0.10 & 0.07 & 0.15 \\
& ins/del & 0.23 & 0.42 & 0.27 & 0.40 & 0.43 & 0.47 & 0.18 & 0.28 \\
& search & 0.07 & 0.05 & 0.08 & 0.08 & 0.05 & 0.08 & 0.08 & 0.12 \\
& delete & 0.07 & 0.17 & 0.08 & 0.15 & 0.20 & 0.20 & 0.05 & 0.07 \\
\hline \multirow{5}{*}{50,000} & insert & 1.15 & 1.58 & 1.12 & 1.85 & 1.12 & 1.30 & 0.67 & 1.35 \\
& search & 0.42 & 0.42 & 0.43 & 0.40 & 0.30 & 0.53 & 0.38 & 0.82 \\
& ins/del & 1.28 & 2.75 & 1.57 & 2.57 & 2.37 & 3.02 & 0.92 & 1.40 \\
& search & 0.40 & 0.42 & 0.42 & 0.48 & 0.30 & 0.53 & 0.40 & 0.75 \\
& delete & 0.38 & 0.95 & 0.55 & 0.93 & 0.97 & 1.15 & 0.33 & 0.35 \\
\hline \multirow{5}{*}{100,000} & insert & 1.77 & 3.23 & 2.12 & 3.35 & 2.77 & 3.13 & 1.17 & 2.42 \\
& search & 0.90 & 0.87 & 0.90 & 0.88 & 0.63 & 1.12 & 0.92 & 1.70 \\
& ins/del & 3.00 & 6.00 & 3.42 & 5.38 & 5.13 & 6.32 & 1.92 & 3.22 \\
& search & 0.97 & 0.92 & 0.88 & 0.98 & 0.63 & 1.12 & 0.82 & 1.70 \\
& delete & 0.87 & 2.08 & 1.17 & 2.05 & 2.10 & 2.40 & 0.70 & 0.67 \\
\hline \multirow{5}{*}{200,000} & insert & 3.92 & 6.42 & 4.27 & 7.25 & 4.92 & 6.03 & 2.58 & 4.93 \\
& search & 1.92 & 1.87 & 1.92 & 1.88 & 1.32 & 2.40 & 1.85 & 3.87 \\
& ins/del & 5.78 & 13.80 & 7.33 & 11.88 & 10.93 & 13.72 & 3.75 & 6.67 \\
& search & 1.90 & 1.93 & 1.92 & 2.13 & 1.33 & 2.38 & 1.75 & 3.97 \\
& delete & 1.67 & 4.55 & 2.48 & 4.45 & 4.43 & 5.10 & 1.40 & 1.35 \\
\hline \hline
\end{tabular}

Time Unit : sec

Table 16: Run time on ordered real inputs 


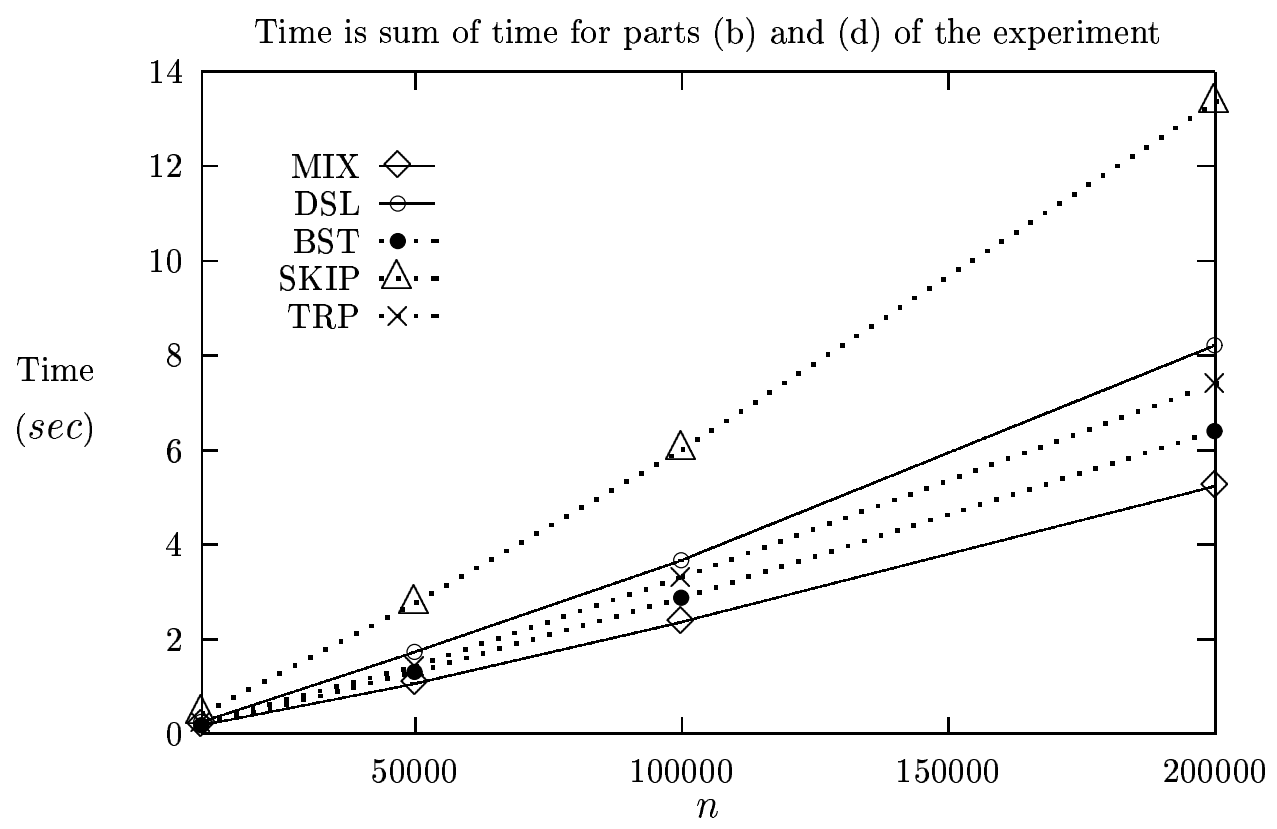

Figure 13: Run time on random real inputs

Time is sum of time for parts (b) and (d) of the experiment

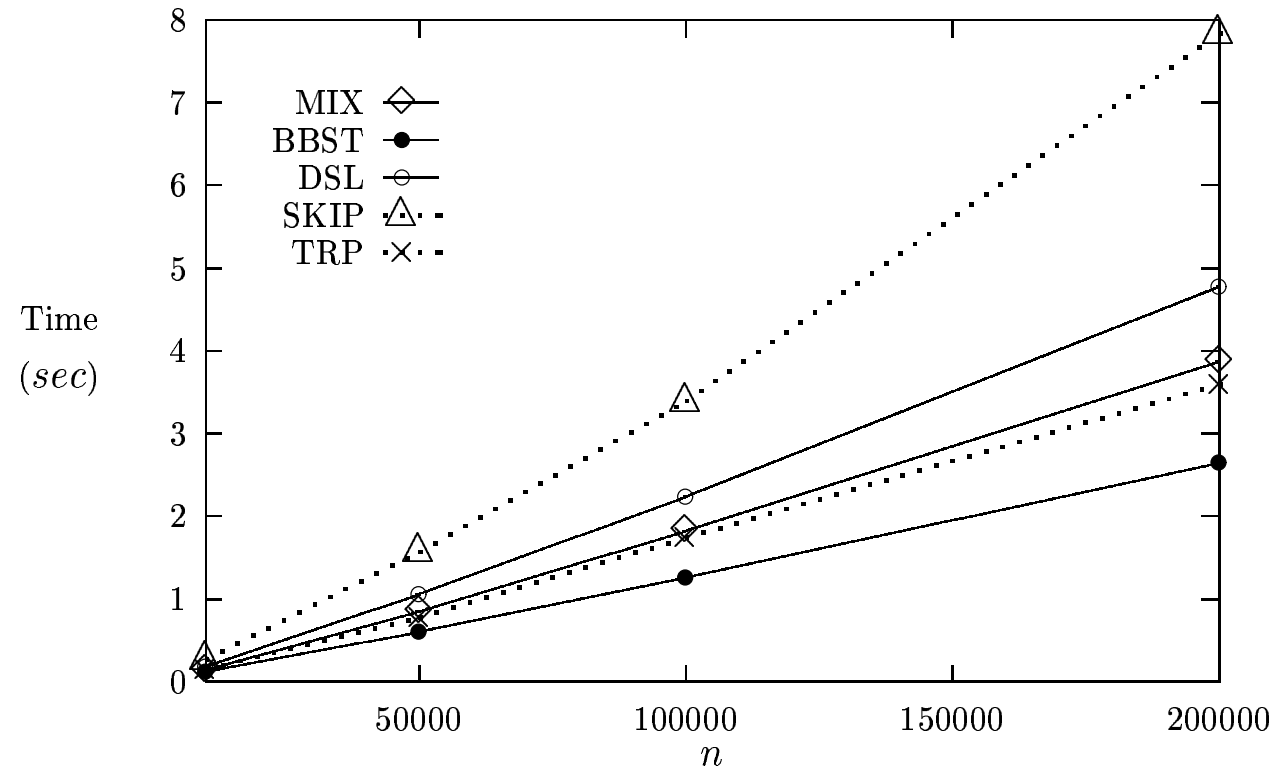

Figure 14: Run time on ordered real inputs 


\section{Conclusion}

We have developed a new weight balanced data structure called $\beta$-BBST. This was developed for the representation of a dictionary. In developing the insert/delete algorithms, we sought to minimize the search cost of the resulting tree. Our experimental results show that BBSTs generally have the best search cost (as measured by the number of comparisons) of the structures considered. Furthermore, this translates into reduced search time when the key comparison cost is relatively high (e.g., for real keys). The insert and delete algorithms for $\beta$-BBSTs are not as efficient as those for other dictionary structures (such as AVL trees). As a result, we recommend $\beta$-BBSTs for environments where searches are done with much greater frequency than inserts and/or deletes. Based on our experiments, we conclude that AVL trees are the best dictionary structure for general applications. We note, however, that this conclusion is based using our $\mathrm{C}$ language simulated pointer implementations on a SUN station. Since run time is quite sensitive to coding practice, choice of programming language, compiler technology, and architectural characteristics such as cache size and cache management policy and since the run times of many of the programmed methods is quite competitive, the relative performance of the data structures considered in this paper could be different when any of the aformentioned factors is changed.

In comparing the different dictionary data structures, we have excluded a comparison of their space requirements. All our binary tree structures require the same number of data and pointer fields. The additional overhead is 1 bit per node for red-black trees, 2 bits per node for AVL-trees, and 4 bytes per node for WB and BBST trees (assuming a four byte integer is used for the weight of a node). So WB and BBST trees have a much larger space overhead than do either red-black or AVL trees.

We have also proposed two simplified versions of the BBST called SBBST and BBSTD. The SBBST seeks only to provide logarithmic run time per operation and unlike the general BBST, does not reduce search cost at every opportunity. The SBBST provides slightly better balance than provided by $\mathrm{WB}(\alpha)$ trees. The BBSTD does not attempt to maintain $\beta$-balance. However it performs rotations to reduce search cost whenever possible. Both versions are very competitive with BBSTs. The SBBST exhibited much better run time performance than BBSTs on random data and the BBSTD slightly outperformed the BBST on ordered data. However, BBSTs generated trees with the lowest search cost (though not by much). 


\section{References}

[ARAG89] C. R. Aragon and R. G. Seidel, Randomized Search Trees, Proc. 30th Ann. IEEE Symposium on Foundations of Computer Science, pp. 540-545, October 1989.

[AVL62] Adel'son-Vel'skii, G. M. and Landis, E. M., An algorithm for the organization of information, Doklady Akad. Nauk., USSR, 146, 2, pp. 263-266, 1962.

[BLUM80] N. Blum and K. Mehlhorn, On the Average Number of Rebalancing Operations in Weight-balanced Trees, Theoretical Computer Science, vol 11, pp.303-320, 1980.

[GUIB78] L. J. Guibas and R. Sedgewick, A Dichromatic Framework for Balanced Trees, Proc. 19th FOCS, pp. 8-21, 1978.

[KARL76] P. L. Karlton, S. H. Fuller, R. E. Scroggs, and E. B. Kaehler, Performance of height-balanced trees, Communications of the ACM, 19, 1, pp. 23-28, 1976.

[MUNR92] J. I. Munro, T. Papadakis and R. Sedgewick, Deterministic Skip Lists, 3rd Annual ACM-SIAM Symposium on Discrete Algorithms, pp. 367-375, January 1992.

[NIEV73] J. Nievergelt and E. M. Reingold, Binary Search Trees of Bounded Balance, SIAM J. Computing, Vol. 2, No. 2, pp. 33-43, March 1973.

[PAPA93] T. Papadakis, Skip Lists and Probabilistic Analysis of Algorithms, PhD Dissertation, Univ. of Waterloo, 1993.

[PUGH90] W. Pugh, Skip Lists: a Probabilistic Alternative to Balanced Trees, Communications of the ACM, vol. 33, no. 6, pp.668-676, 1990.

[SAHN98] S. Sahni, Data Structures, Algorithms, and Applications in C++, New York: WCB McGraw-Hill, 1998.

[SEDG94] R. Sedgewick, Algorithms in C++, Mass.: Addison-Wesley Pub. Co., 1994.

[TARJ83] R. E. Tarjan, Updating a Balanced Search Tree in O(1) Rotations, Information Processing Letters, Vol. 16, pp. 253-257, June 1983. 